\title{
EUV-induced hydrogen plasma: pulsed mode operation and confinement in scanner
}

\author{
Mark van de Kerkhof $\odot,{ }^{a, b}, *$ Andrei M. Yakunin, ${ }^{\text {a }}$ Dmitry Astakhov, ${ }^{\text {c,d }}$ \\ Maarten van Kampen, ${ }^{a}$ Ruud van der Horst, ${ }^{a}$ and Vadim Banine ${ }^{\mathrm{a}, \mathrm{b}}$ \\ ${ }^{a} A$ SML Netherlands B.V., Veldhoven, The Netherlands \\ ${ }^{b}$ Eindhoven University of Technology, Department of Applied Physics, Eindhoven, \\ The Netherlands \\ 'ISTEQ B.V., Eindhoven, The Netherlands \\ ${ }^{\mathrm{d}}$ Institute for Spectroscopy of the Russian Academy of Sciences, Moscow, Russia
}

\begin{abstract}
In recent years, EUV lithography scanner systems have entered high-volume manufacturing for state-of-the-art integrated circuits, with critical dimensions down to $10 \mathrm{~nm}$. This technology uses 13.5-nm EUV radiation, which is shaped and transmitted through a near-vacuum $\mathrm{H}_{2}$ background gas. This gas is excited into a low-density $\mathrm{H}_{2}$ plasma by the EUV radiation, as generated in pulsed mode operation by the laser-produced plasma in the EUV source. Thus, in the confinement created by the walls and mirrors within the scanner system, a reductive plasma environment is created that must be understood in detail to maximize mirror transmission over the lifetime and to minimize molecular and particle contamination in the scanner. In addition to the irradiated mirrors, reticle, and wafer, the plasma and radical load to the surrounding construction materials also must be considered. We provide an overview of the EUV-induced plasma in the scanner context. Special attention is given to the plasma parameters in a confined geometry, such as that found in the scanner area near the reticle. It is shown that plasma confinement and resulting contributions from secondary electron emission delay the formation of the plasma sheath and thereby reduce the peak ion energies to below the sputtering threshold for mirrors and construction materials. Furthermore, for a confined pulsed plasma with a pulse period shorter than the decay time of the plasma, the plasma consists of a quasi-steady-state cold background plasma and periodic transient peaks in ion energy and ion flux. In terms of modeling, this means that no assumptions can be made on the electron distribution functions and a (Monte-Carlo) particle-in-cell (PIC) model is needed. We present an extension of the PIC model approach to complex three-dimensional geometries and to multiple pulses using a hybrid PIC-diffusion approach. Also, the translation of these specific plasma parameters to off-line setups and the aspects that must be included to make a meaningful translation from off-line laboratory EUV setups to the scanner plasma are discussed. (c) The Authors. Published by SPIE under a Creative Commons Attribution 4.0 Unported License. Distribution or reproduction of this work in whole or in part requires full attribution of the original publication, including its DOI. [DOI: 10.1117/1.JMM.20.3.033801]
\end{abstract}

Keywords: lithography; EUV; EUV-induced plasma; pulsed discharge; particle-in-cell; hybrid PIC.

Paper 21044 received May 10, 2021; accepted for publication Aug. 2, 2021; published online Aug. 16, 2021.

\section{Introduction}

EUV lithography has established itself as the technology of choice for high-volume manufacturing (HVM) of 5-nm node and beyond, ensuring that Moore's law will continue for the coming years. ${ }^{1}$ This technology uses 13.5 -nm EUV radiation, which is generated in pulsed mode operation by a laser-produced plasma (LPP) in the EUV source. Even with the outstanding imaging and overlay capability of the current EUV scanners, ${ }^{2}$ device output and yield can still be affected adversely by other factors, such as molecular or particulate contamination on critical imaging surfaces. ${ }^{3}$ Also, high source power and mirror reflectivity must be secured over the full scanner

*Address all correspondence to Mark van de Kerkhof, mark.van.de.kerkhof-msd@asml.com 


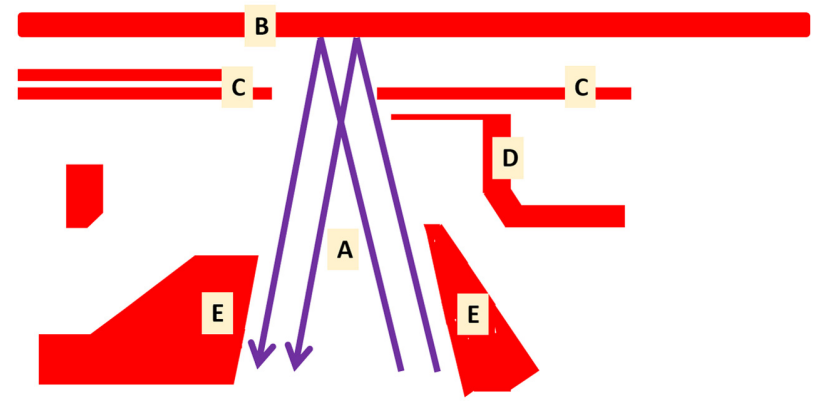

Fig. 1 Schematic of scanner RME, (A) EUV beam, (B) reticle surface, (C) reticle masking blades, (D) uniformity correction fingers, and $(E)$ beam confinement.

lifetime. Theoretically, it would be ideal to do EUV lithography in vacuum conditions since EUV photons are absorbed by any medium. However, in practice, a background gas of roughly $5 \mathrm{~Pa}$ hydrogen $\left(\mathrm{H}_{2}\right)$ must be used to maintain self-cleaning conditions for the sensitive EUV mirrors. Hydrogen was chosen as the background gas because of the low EUV-absorption and high chemical activity of H-radicals and ions. ${ }^{4}$ This gas is excited into a low-density $\mathrm{H}_{2}$ plasma by the EUV radiation. A detailed understanding of the EUV-induced plasma is crucial, as this creates a highly aggressive environment for both the mirrors and the surrounding construction materials. In the past 25 years, main focus has been on the interactions of EUV and plasma with the mirror surfaces facing the EUV and EUV plasma. ${ }^{5}$ However, for molecular and particle contamination control, the interaction of plasma with the construction and functional materials close to the beam must be optimized.

The EUV beam will vary in intensity and shape throughout the scanner, as will the plasma that is induced by this ionizing radiation. Although most of the described physical mechanisms are generic for any radiation-induced plasma, this paper zooms in on the specific case of a confined plasma, meaning that the dimensions of the walls confining the plasma are of same order of magnitude as the mean-free path lengths of the electrons and ions. As a specific example, the plasma details of the so-called reticle mini-environment (RME, see Fig. 1) are worked out in detail. This area is of particular interest for the plasma-reticle interactions and particle contamination control. ${ }^{3}$ Mutatis mutandis, the same underlying physics is applied to other areas of the scanner.

Section 2 describes the EUV generation, and Sec. 3 details the EUV-induced plasma. Section 4 presents the specifics of the RME plasma, and Sec. 5 briefly discusses the implications to off-line set-ups to emulate the EUV-induced scanner plasma.

\section{EUV Generation}

EUV light is commercially generated from a hot plasma emitting light incoherently over a large solid angle. The dominant EUV source technology is LPP, in which pulsed bursts of EUV are emitted by a tin ( $\mathrm{Sn}$ ) plasma that is created by an intense pulsed IR laser. The current generation of LPP EUV sources uses a pre-pulse (PP) laser focused onto a stream of liquid Sn droplets. The interaction of the PP laser with each tin droplet causes the droplet to deform into a disk-like target with a reduced thickness that is more favorable for EUV production and has a reduced selfabsorption. As the tin target is formed, a high-power $(>20 \mathrm{~kW}), 10.6 \mu \mathrm{m}$ wavelength short-pulse $\mathrm{CO}_{2}$ laser main pulse beam is used to rapidly heat and ionize the disk-like Sn target. ${ }^{6}$ An intensely hot plasma with a temperature of several tens of $\mathrm{eV}$ is generated; it efficiently emits EUV radiation at the primary resonances of multiply ionized $\mathrm{Sn}$ around $13.5 \mathrm{~nm},{ }^{7}$ plus secondary peaks in the VUV and UV, and broadband blackbody radiation in accordance with Wien's classical law. ${ }^{89}$ However, this spectrum is filtered by the series of narrowband Bragg-reflection mirrors in the scanner, so the photon spectrum inside the scanner at the reticle and wafer level may be assumed to be $13.5 \mathrm{~nm}( \pm 1.5 \%),{ }^{10}$ as shown in Fig. 2 . This is in contrast to many laboratory setups, which are often based on EUV sources using grazing incidence collector optics without inherent spectral filtering. 

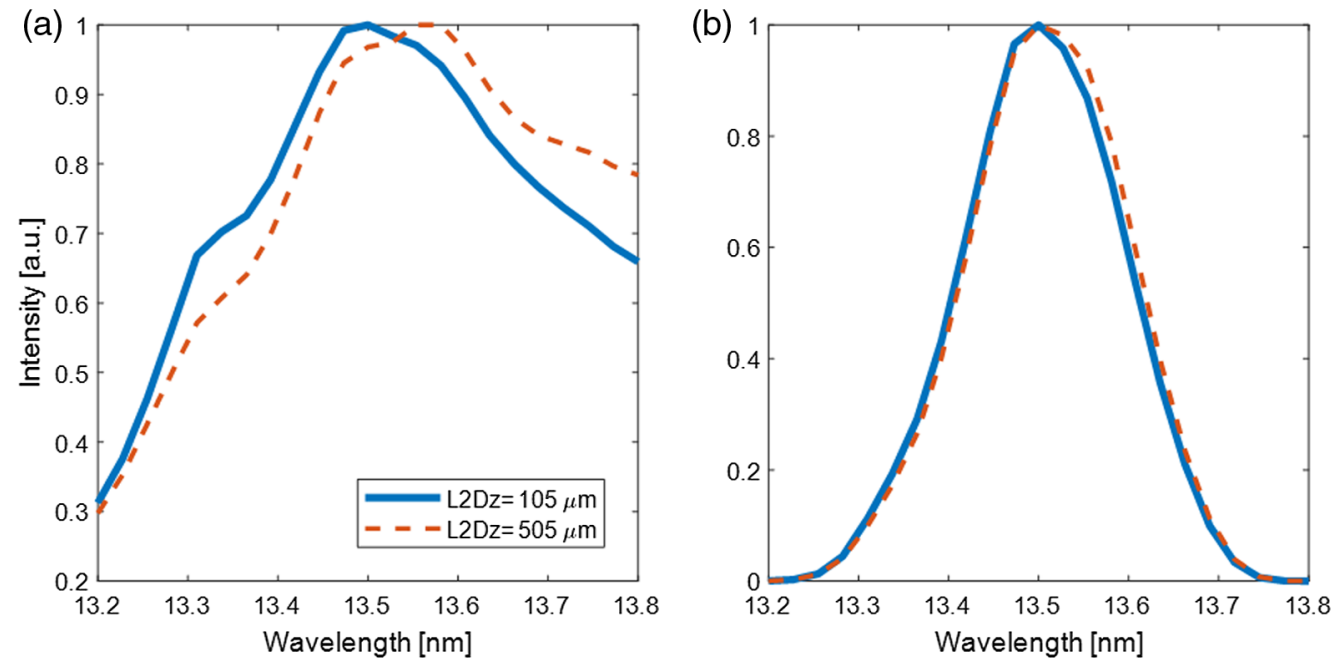

Fig. 2 (a) Measured LPP EUV source output spectrum for nominal (labeled L2Dz = $105 \mu \mathrm{m}$ ) and detuned laser-to-droplet targeting conditions (labeled L2Dz $=505 \mu \mathrm{m}$ ) (meaning the irradiating laser-to-droplet focus is off by $400 \mu \mathrm{m}$; (b) same targeting conditions, after filtering by the narrowband Bragg-reflection mirrors in the scanner. ${ }^{11}$

In addition to EUV, VUV and UV are emitted as blackbody radiation and lower ionization states. UV is generated mainly in the cooler parts of the Sn plasma (in the range of around 2 to $20 \mathrm{eV}$ ) and during cooling-down of the Sn plasma. The ionizing VUV part of the spectrum is effectively absorbed by the source background gas ${ }^{12}$ and the narrowband Bragg mirrors. ${ }^{13} \mathrm{UV}$ wavelengths between roughly 130 and $250 \mathrm{~nm}$, however, travel through the scanner without only limited attenuation relative to the EUV. For a properly optimized Sn plasma, the UV contribution is in the order of $\sim 1 \%$ and may be ignored during the EUV pulse. However, the different temporal behavior might be relevant since the UV afterglow may persist for some fraction of a microseconds during the cooling-down and recombination phase of the Sn plasma. Indeed, significant UV afterglow has been measured for extended solid-state targets, ${ }^{14}$ but for mass-limited droplet targets this should be (much) smaller since the expanding Sn plasma is not focused into the scanner. Unfortunately, no time-resolved spectrometer setup exists today to measure this for the current LPP source. From depth of focus and ion velocity estimates, the duration of the UV afterglow may be estimated to be $\sim 0.2$ to $0.3 \mu \mathrm{s}$. Even at $\sim 1 \%$ of the EUV intensity, the UV afterglow might significantly influence the scanner plasma by efficient photoemission of relatively cold electrons from the surfaces around the EUV beam.

The LPP source is highly transient, firing short $<100 \mathrm{~ns}$ pulses with energy of $\sim 5 \mathrm{~mJ}$ at a repetition period of $20 \mu \mathrm{s}$, with the peak of EUV in the first $50 \mathrm{~ns}$ and a tail of broadband radiation; see Fig. 3. This equates to a frequency of $50 \mathrm{kHz}$ and average output of $250 \mathrm{~W}$. The typical

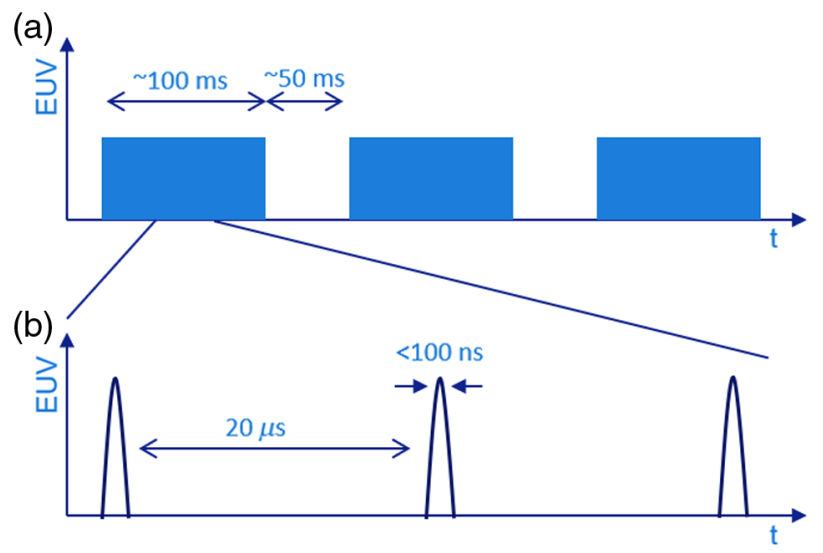

Fig. 3 (a) Sketch of EUV pulse train and (b) individual pulses. 
Table 1 EUV beam properties at RME for 250W LPP source.

\begin{tabular}{lc}
\hline \hline Parameter & Value \\
\hline EUV power at reticle & $50 \mathrm{~W}$ \\
Beam dimensions & $11 \times 1 \mathrm{~cm}^{2}$ \\
Photon flux & $\sim 10^{21} \mathrm{~s}^{-1} \cdot \mathrm{m}^{-2}$ \\
Photon energy & $92 \mathrm{eV}( \pm 1 \mathrm{eV})$ \\
Pulse frequency & $50 \mathrm{kHz}$ \\
Pulse length & $<100 \mathrm{~ns}$ \\
\hline \hline
\end{tabular}

EUV pulse train duration is in the order of $\sim 100 \mathrm{~ms}$, or $\sim 5000$ pulses, and is stopped for several milliseconds after every scanning die exposure (whereas the wafer stage is stepped to the next exposure position).

At the reticle level, the effective EUV power is $50 \mathrm{~W}$, and the EUV beam is essentially rectangular in shape (actually the rectangle is somewhat curved ${ }^{15}$ ), with a slit width of about $11 \mathrm{~cm}$ and scanning width of about $1 \mathrm{~cm}$. The intensity distribution may be considered uniform in the slit direction and roughly Gaussian in the scanning direction. The main EUV properties for a 250-W LPP source at RME are summarized in Table 1.

\section{EUV-Induced Scanner Plasma}

Photoionization by the high-frequency pulsed EUV results in repeating cycles of plasma generation, expansion, cooling, and recombination, with sharp transients around the short $<100 \mathrm{~ns}$ EUV pulse and a repetition period of $20 \mu \mathrm{s}$. Electrons are created by ionization of gas molecules, the photoelectric effect, and secondary electron emission (SEE) from plasma-facing surfaces, and they are lost by absorption at these surfaces. Ions are created by ionization of gas molecules and lost by recombination at the walls. At higher ionization degrees, volume recombination also results in loss of ions and electrons. Several phases can be distinguished (transition moments are indicative and depend on pressure, power, and plasma geometry), as outlined in Fig. 4:

- 0 to $0.2 \mu$ s photoionization and photoelectric electrons;

- 0.2 to $1 \mu$ s secondary ionization, electron exchange at walls, fast electron cooling, and fast expansion;

- 1 to $20 \mu$ s ambipolar diffusion, decay, and slow electron cooling;

- $20 \mu$ s next EUV pulse: repeat.

These phases are described in more detail in the following sections.

(a)

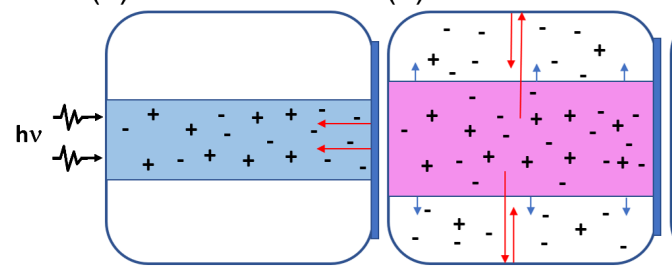

(c)

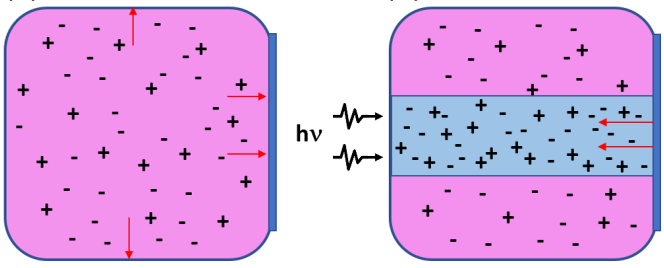

Fig. 4 Phases of pulsed EUV-induced hydrogen plasma, (a) primary ionization and photoelectric effect, (b) expansion and electron exchange, (c) diffusion and decay, and (d) next EUV pulse. Red arrows indicate main electron fluxes to and from mirror surface (on the right) and other walls. 


\subsection{Plasma Equations}

The definition of a plasma is a quasineutral ionized gas governed by the strong interaction between electrons and ions and by the different thermodynamical properties of each species. This state may be created by, e.g., electromagnetic fields, microwaves, or discharges or by ionizing radiation such as EUV. A textbook plasma is in local thermal equilibrium (LTE), and the electron energy distribution is Maxwellian. Based on these assumptions, standard equations can be found for the most important plasma parameters: temperature, Debye length, plasma frequency, sheath potential, and sheath electrical field. As we will see, the pulsed EUV-induced plasma is strongly transient and typically is not in LTE, and the electron energy distribution is not Maxwellian. ${ }^{16}$ This in turn means that many classical plasma assumptions will not, or will not always, apply, and care must be taken with the standard equations for Debye length and plasma sheath. ${ }^{17}$ Still, the resulting quasisteady-state background plasma can often be approximated well enough in classical terms, even if the transient peaks may deviate from this.

The Debye electrical shielding length is given as

$$
\lambda_{\mathrm{D}}=\sqrt{\frac{\epsilon_{0} T_{\mathrm{e}}}{n_{\mathrm{e}} e}},
$$

where $\epsilon_{0}$ is the permittivity of free space, $k_{\mathrm{B}}$ is the Boltzmann constant, $e$ is the elementary charge, $T_{e}$ is the electron temperature in $\mathrm{eV}$, and $n_{e}$ is the electron density.

The characteristic time scale of Debye shielding by electrons is the inverse of the plasma electron frequency $\omega_{\mathrm{pe}}=\sqrt{e^{2} n_{e} / \epsilon_{0} m_{e}}$, and the plasma ion frequency is $\omega_{\mathrm{pi}}=\sqrt{e^{2} n_{i} / \epsilon_{0} m_{i}}$. Typical values for EUV-induced scanner plasma are $\lambda_{\mathrm{D}} \cong 0.1 \mathrm{~mm}, \omega_{\mathrm{pe}} \cong 10^{9} \mathrm{~s}^{-1}$, and $\omega_{\mathrm{pi}} \cong 10^{7} \mathrm{~s}^{-1}$, giving a plasma response time of $\sim 0.1 \mu \mathrm{s}$.

In the bulk of the plasma, the coulomb forces between electrons and ions prevent charge separation beyond the Debye length, maintaining quasineutrality and a negligible electrical field. However, at the plasma-wall interface, the higher mobility of the electrons leads to a sheath region where the density of electrons is smaller than the density of ions. Thus, a space charge region is formed near the wall, which attracts ions until an equilibrium is reached, as shown in Fig. 5. The resulting potential distribution and electric field in the sheath are determined by the local plasma density and electron temperature. The sheath width for an unbiased wall is a few Debye lengths and may be approximated as

$$
L_{s} \cong 3 \cdot \lambda_{\mathrm{D}}=3 \cdot \sqrt{\frac{\epsilon_{0} T_{\mathrm{e}}}{n_{\mathrm{e}} e}} .
$$

A typical value for the sheath width in scanner is $\sim 0.3 \mathrm{~mm}$. The plasma-to-wall potential $\phi_{p}$ is closely linked to the electron temperature (combining sheath potential with the presheath Bohm potential):

$$
\phi_{p}=-T_{e} \ln \left(\frac{m_{i}}{2 \pi m_{e}}\right)^{1 / 2}-\frac{1}{2} T_{e}
$$
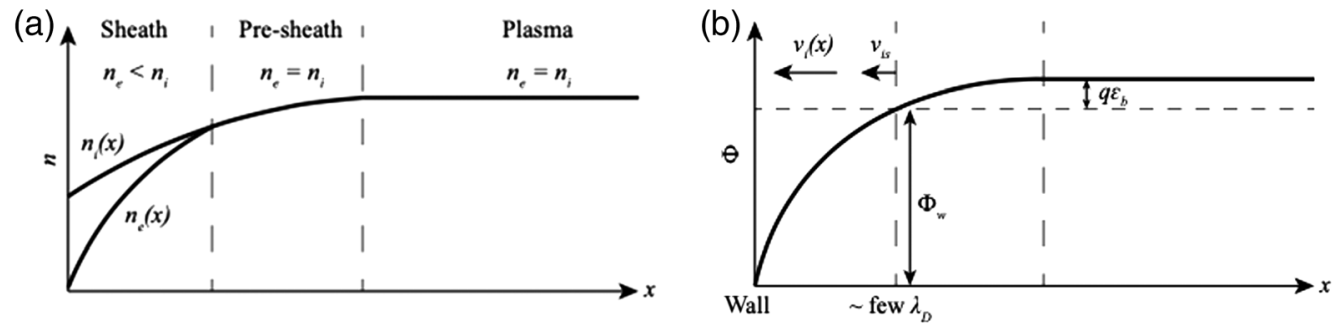

Fig. 5 Illustration of the sheath, (a) the ion and electron densities and (b) the potential distribution and the resulting ion acceleration. From Ref. 18. 
in which the sheath potential and presheath Bohm potential $\Phi_{b}=\frac{1}{2} T_{e}$ have been combined. ${ }^{16}$ Hydrogen, with $\mathrm{H}_{3}^{+}$as the dominant ion $\left(m_{i}=3 \mathrm{amu}\right)$ leads to a plasma-wall potential $\phi_{p} \cong 4 T_{e}$. Typical scanner pressure is $\sim 5 \mathrm{~Pa}$, so no collisions take place across the narrow sheath and the ion is accelerated to a kinetic energy at impact of

$$
U_{\text {ion }}=e \cdot \phi_{p} \cong 4 \cdot T_{e} .
$$

For the afterglow plasma with $T_{e} \sim 0.1 \mathrm{eV}$, this gives a first-order estimate of $\sim 1 \mathrm{eV}$ for the ion energy. While the plasma potential and ion energy are low during the afterglow in between pulses, they may reach significant values in the transients during and after the EUV pulse. All ions entering the sheath from the presheath are swiftly accelerated toward the surface by the electrical sheath field. The ion flux to the wall $\varphi_{i, w}$ is the product of the local ion density in the presheath $n_{i, \mathrm{ps}}$ and the Bohm velocity $v_{B}=\sqrt{e \cdot T_{e} / m_{i}}$ :

$$
\varphi_{i, w}=n_{i, \mathrm{ps}} \cdot v_{B}=n_{i, \mathrm{ps}} \cdot \sqrt{T_{e 2}} \cdot \sqrt{e / m_{i}} .
$$

Within the conductive plasma, the electrical field is effectively zero over distances longer than the Debye length, whereas the sheath region shows an exponentially decreasing electrical field $E_{s}$ from a maximum value $\phi_{p}$ at the wall to approximately zero at the sheath edge. Near the wall, the value is approximately

$$
E_{s}(0)=\frac{d \phi(0)}{d x} \cong-\frac{2}{L_{s}} \cdot \phi_{p} \cong-2.5 \cdot \sqrt{\frac{n_{\mathrm{e}} e T_{\mathrm{e}}}{\epsilon_{0}}} .
$$

While the electrical field is negligible in the afterglow, during the transient phase it may reach values of $E_{s}(0) \sim 100 \mathrm{kV} / \mathrm{m}$, as the electron energies may be above $T_{e}>10 \mathrm{eV}$ (and $\lambda_{D} \cong 0.5 \mathrm{~mm}$ ). ${ }^{3}$

For a pulsed plasma, the plasma cools down and diffuses out during the afterglow, but if the pulse period is shorter than the decay time, the next pulse will be a combination of the residual afterglow plasma and the newly created plasma. These two contributions have independent electron energy distributions. The combined electron energy distribution may be approximated by a bi-Maxwellian distribution, in which the plasma potential is typically dominated by the high energy population, with a (downward) correction by the low-energy population equation. Provided the flux of high-energy electrons is higher than the ion flux ${ }^{19}$ Godyak et al. ${ }^{20}$ derived a modification to the sheath potential equation for a bi-Maxwellian distribution:

$$
\phi_{p} \cong-T_{e 2}\left\{4.6-\ln \left(\frac{n_{1}}{n_{2}} \cdot \sqrt{T_{e 1} / T_{e 2}}\right)\right\},
$$

where $T_{e 2}$ and $T_{e 1}$ are the electron temperature of the high- and low-energy populations, respectively, and $n_{2}$ and $n_{1}$ are the corresponding electron densities; the numerical factor 4.6 is specific for hydrogen. ${ }^{19}$ As above, the Bohm presheath potential has been added to the sheath potential. The strong effect of the high-energy population on the sheath potential can be understood by the fact that more energetic electrons will be able to escape the potential well formed by the positive space charge of the plasma, whereas the low-energy electrons are effectively trapped closer to the center of the plasma. Conversely, bulk parameters such as Debye length and diffusion constant will be dominated by the overall average electron energy, which is typically dominated by the larger low-energy electron population. In a radiation-induced plasma, a split electron population might also occur because of the low-energy secondary electron being generated from irradiated surfaces, in addition to the electrons generated in the gas ionization process. In the case of a pulsed radiation-induced plasma, these effects combine to three (or more) electron populations, in which case $T_{e 2}$ should be the temperature associated with the fraction with the highest energy (again with the condition that the flux of this fraction must be higher than the ion flux), and $T_{e 1}$ is the combined average of the other fractions. 


\subsection{Plasma Diagnostics}

The photoelectric currents induced by EUV and EUV-induced fluorescence complicate plasma measurements and diagnostics, rendering Langmuir probes difficult to interpret. ${ }^{15}$ For our measurements of temporally resolved ion fluxes and energies, we use a retarding field energy analyzer (RFEA; type Semion Single Sensor from Impedans ${ }^{21}$ ); extensive details are given by Van de Ven. ${ }^{22}$ Also, the RFEA concept is sensitive to spurious photoelectrons from the internal grids, creating major artifacts during the EUV pulse and directly afterward. For the present work, the RFEA readout electronics and sampling rate were optimized to minimize the "unusable" time interval to $\sim 0.2 \mu \mathrm{s}$.

Species-resolved ion energy distributions have been measured using an electrostatic quadrupole plasma analyzer (EQP1000, Hiden Analytical), an ion mass spectrometer enabling mass, and energy-resolved measurements; details are given by Van de Ven. ${ }^{17}$ The EQP does not provide time-resolution to better than $3 \mu \mathrm{s}$, but it does capture the transient first $\sim 1 \mu \mathrm{s}$ as part of the overall ion energy distribution.

\subsection{Plasma Modeling: Hybrid PIC}

The pulsed photoionization origin of the plasma leads to important differences from textbook plasma, such as strong transients and a non-Maxwellian energy distribution function during and after the EUV pulse (of $<100 \mathrm{~ns}$ ). This precludes the use of fluid-based models, which rely on continuity equations for moments of the distribution functions for electron density, velocities, and energies. Instead, a kinetic model that can solve the full equations for the electron distribution functions without any a priori assumptions about their shapes, such as (Monte-Carlo) particle-in-cell (PIC), must be used. The essence of the PIC model used consists of a Poisson equation solver, followed by updating the charged particles positions and velocities based on the obtained field distribution and individual particle velocities. ${ }^{23}$ This also allows for accurate tracking of the ions, which have mean-free path lengths in the order of mm's in lowpressure $\mathrm{H}_{2}$ and thus experience only a few collisions with neutral gas molecules before hitting a surface. This PIC model has been tailored for simulation of EUV-induced plasma, ${ }^{24}$ and the resulting model has been validated for EUV at a relevant pressure of $5 \mathrm{~Pa}$ in an off-line test setup using an EQP analyzer, ${ }^{25}$ as shown in Fig. 6, showing agreement in general structure and ratio between ion species. It was not possible to reliably convert the EQP count per second into ion flux at the entrance to the EQP, so it was not possible to establish error bars on the comparison between model and experiment.

A drawback of the kinetic PIC-approach is the high computational cost for larger volumes and/or longer timescales. Typical decay times of the scanner plasma at pressures of 1 to $10 \mathrm{~Pa}$ are longer than the pulse interval of $20 \mu \mathrm{s}$, and plasma builds up over several pulses. For these conditions, direct multipulse PIC simulations are still possible, but the run time of such simulations becomes prohibitive, since this is dominated by the simulation of the decay of the cold plasma, and an explicit PIC model needs to run with mesh cell size of few Debye radii and a time step $\Delta t$ smaller than the inverse plasma electron frequency, or $\Delta t<1 / \omega_{\mathrm{pe}}=0.5 \mathrm{~ns}$ for a $250-\mathrm{W}$ EUV source (it should be noted that for the decay of the cold EUV-induced plasma, these conditions are typically even more restrictive than for the hot phase during EUV pulse, since the electron temperature of the plasma decreases faster than the ion and electron density).

To resolve this, a dedicated hybrid three-dimensional (3D)-PIC model was developed; it uses a rigorous kinetic model for the initial non-Maxwellian phase during and directly after the EUVpulse, up to $\sim 2 \mu \mathrm{s}$, and a fast fluid-like drift-diffusion model for the electron density and energies in the subsequent diffusion phase, when the deviation from a Maxwellian energy distribution becomes small enough to ignore. The transition criterium was taken to be when the electron high energy tail dropped below $8 \mathrm{eV}$, in which case all electronic energy loss processes except vibrational and rotational processes may be neglected, and all remaining reaction rates are accurately approximated by a Maxwellian distribution (of $T_{e}<1 \mathrm{eV}$ ). The ions are still modeled kinetically, which is needed because of the lack of collisions. Focus of the code development was the (unstructured) meshing complexities of the relevant 3D geometries, with many slits, electrically floating surfaces, and dielectric surfaces. Such a hybrid model allows for significantly 


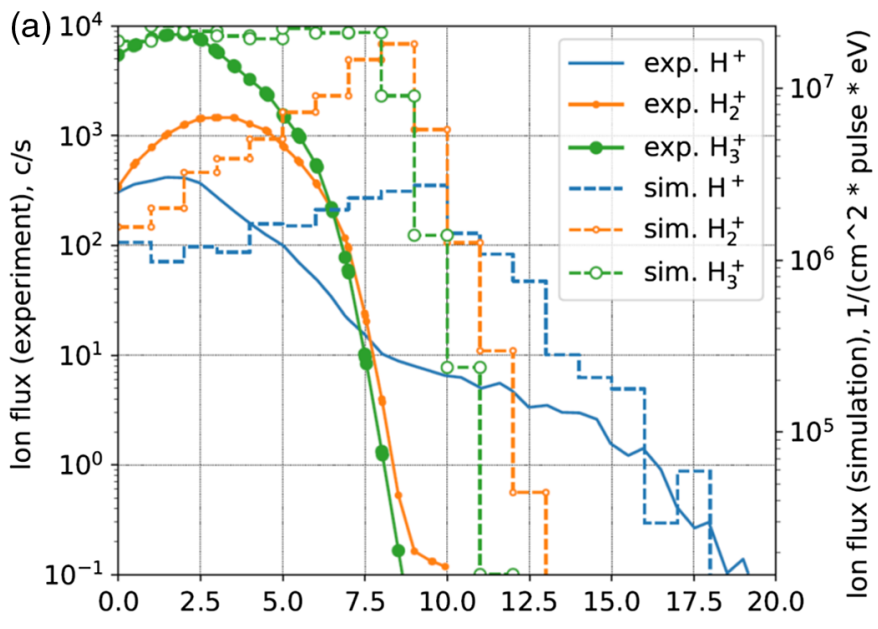

(b)

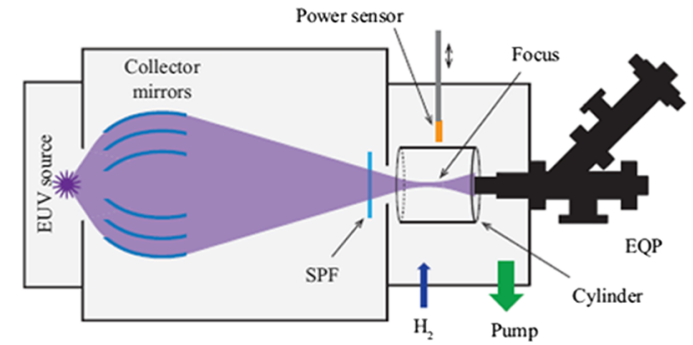

Fig. 6 EUV-induced ion energy density functions, as simulated by PIC model and measured by (a) EQP in off-line (b) EUV set-up. ${ }^{17}$

accelerating multipulse simulations ${ }^{26}$ since the evolution of the cold afterglow plasma between EUV pulses now needs to be described only with resolution of ion time scales (in the order of $\tau_{i}=30 \mathrm{~ns}$ for $5 \mathrm{~Pa}$ and $250 \mathrm{~W}$ EUV source).

Figure 7 shows the switching moment from full PIC to hybrid mode as well as the gain in model performance. The overall performance gain in temporal hybrid mode is $\sim 4 \times$ over a full pulse cycle of $20 \mu \mathrm{s}$, with further runtime reduction potential identified in coarser meshing. The general validity of such an approach for hydrogen pressures around $5 \mathrm{~Pa}$ has been validated by Van de Ven, ${ }^{17}$ as shown in Fig. 7(c).

This approach also enables modeling of multiple pulses. As the hybrid model reaches the time of the next EUV pulse, the drift-diffusion model is converted back to be starting point for the next iteration of numerical particle-in-cell, maintaining the obtained spatial and average electron energy distributions. Subsequently, the next EUV pulse is added to this distribution, and the next model iteration starts. During this next EUV pulse, the energetic photoelectrons and associated ions are added to the existing colder populations of electrons and ions. Pulse by pulse, the electron density build up until equilibrium is reached with diffusion losses to the walls and volume recombination, which depends on the confinement geometry, resulting in a

(a)

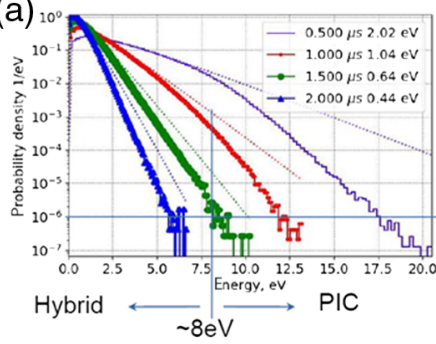

(b)

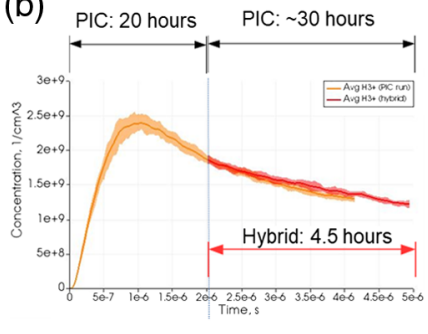

(c)

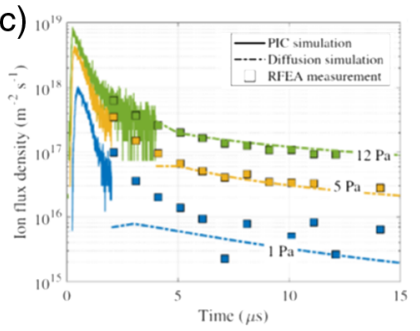

Fig. 7 (a) 3D PIC-hybrid switch condition (dotted lines are Maxwellian fits). (b) Reduction of simulation time by hybrid model for first $5 \mu \mathrm{s}$. (c) Validation of hybrid model approach by RFEA. ${ }^{17}$ 

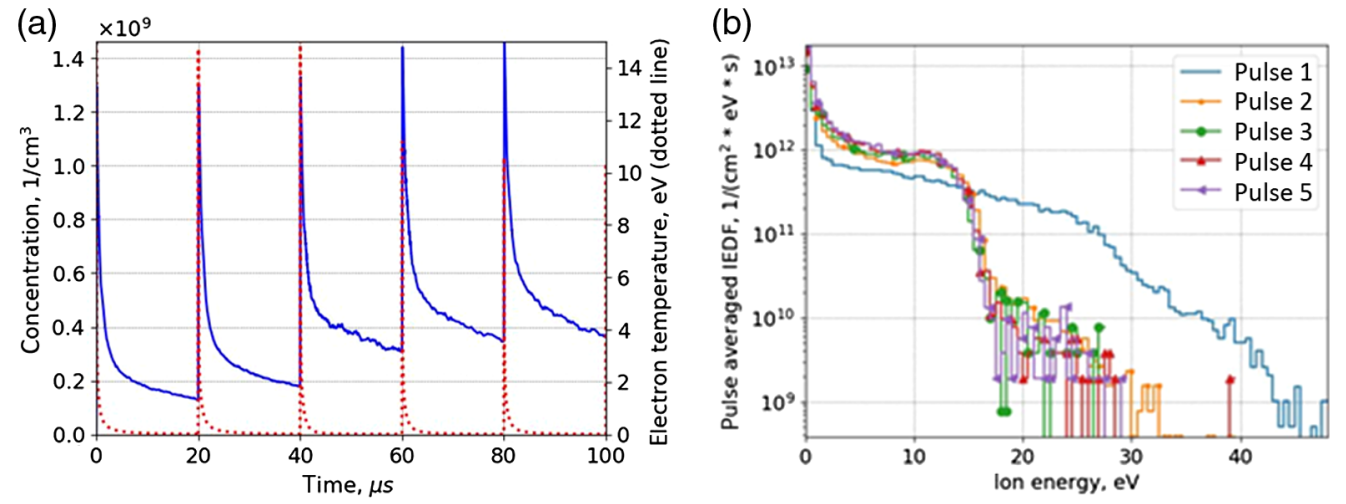

Fig. 8 (a) Example of hybrid PIC modeling of evolution of electron density and electron temperature in a pulsed plasma, within the EUV beam below the reticle. Build-up of stable background with a few pulses can be clearly observed. (b) Evolution of pulse-averaged IEDF at the edge of the beam.

quasisteady-state background with transient peaks in electron temperature, ion density, and ion flux for each EUV pulse. For an open plasma geometry, this equilibrium might take hundreds of pulses to reach, whereas for a confined geometry such as the scanner RME, this might be reached within a few pulses, as shown in Fig. 8.

\subsection{EUV-Induced Plasma}

\subsubsection{Plasma generation}

The 92-eV EUV photons lead to photoionization of the hydrogen background gas. The total cross section for photo-ionization of hydrogen for $92 \mathrm{eV}$ photons is $\sigma_{h \nu}=6.2 \cdot 10^{-24} \mathrm{~m}^{2}$ and gas density at room temperature and a pressure of $5 \mathrm{~Pa}$ is $n_{\mathrm{H} 2}=1.21 \cdot 10^{21} \mathrm{~m}^{-3}$, resulting in an attenuation length of $l=\left(n_{\mathrm{H} 2} \sigma_{h \nu}\right)^{-1}=0.13 \mathrm{~km} .{ }^{27}$ This is much larger than the plasma dimensions within the scanner, so the initial electron and ion production per unit volume $n_{h \nu}$ from photon-ionization can be estimated using a linear approximation of the Beer-Lambert law of absorption:

$$
\frac{d n_{h \nu}}{d t}=\frac{P_{\mathrm{euv}}}{h \nu} \cdot n_{\mathrm{H} 2} \cdot \sigma_{h \nu}
$$

where $P_{\text {euv }}$ is the EUV power (and $\varphi_{h \nu}=P_{\text {euv }} / h \nu$ is the average photon flux density). Assuming a 250 -W EUV source and a local EUV power of $\sim 50 \mathrm{~W}$ at the regions of interest in the scanner, at a pressure of around $5 \mathrm{~Pa}$, the initial hydrogen ion production is in the order of $10^{19} \mathrm{~m}^{-3} \mathrm{~s}^{-1}$. Taking lateral beam dimensions of $\sim 11 \times 1 \mathrm{~cm}^{2}$, the average ion flux is in the order of $10^{17} \mathrm{~m}^{-2} \mathrm{~s}^{-1}$. At $50-\mathrm{kHz}$ source frequency, the integrated initial ion density over a single pulse is in the order of $10^{15} \mathrm{~m}^{-3} \mathrm{~s}^{-1}$, and the plasma ionization degree is in the order of $10^{-4} \%$.

The dominant photoionization mechanisms for molecular hydrogen are nondissociative ( $\sim 80 \%$ branching ratio) and dissociative single ionization $\left(\sim 15 \%\right.$ branching ratio): ${ }^{28,29}$

$$
\begin{gathered}
h \nu+\mathrm{H}_{2} \rightarrow \mathrm{H}_{2}^{+}+e^{-}, \\
h \nu+H_{2} \rightarrow \mathrm{H}^{+}+\mathrm{H}+e^{-}, \\
h \nu+\mathrm{H}_{2} \rightarrow 2 \mathrm{H}^{+}+2 e^{-} .
\end{gathered}
$$

The ionization energy of hydrogen is $15.4 \mathrm{eV},^{30}$ and for nondissociative photoionization, the large excess energy of $76 \mathrm{eV}$ is divided over the resulting electron and ion. Momentum conservation and the large ratio in mass between ion and electron dictate that the excess energy for nondissociative ionization is carried for $>99.9 \%$ by the photoelectron, whereas the ions remain 


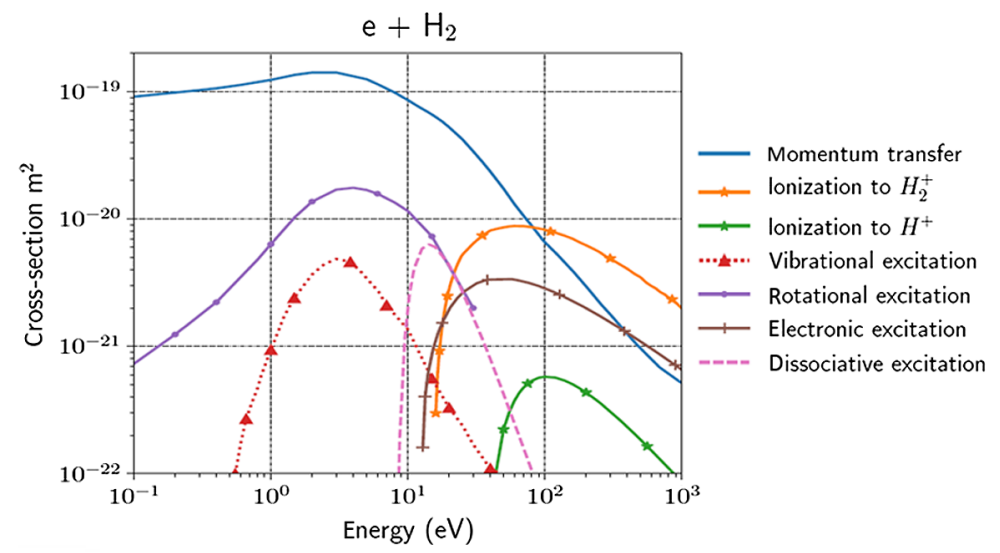

Fig. 9 Cross sections of possible electron-neutral collisions. Based on data from Refs. 33 and 32.

at roughly twice room temperature. ${ }^{31}$ For dissociative ionizations, the energy is more evenly distributed with the photoelectron carrying 60 to $70 \mathrm{eV}$ and the ions and radicals each carrying in the order of $10 \mathrm{eV}^{32}$ In this process, the radical atom may end up in an excited electronic state, increasing its energy further. The resulting plasma thus contains slow $\mathrm{H}_{2}^{+}$and fast $\mathrm{H}^{+}$ions and fast radicals.

The peak plasma and radical densities increase further by secondary electron-impact ionizations by the energetic photoelectrons:

$$
\begin{gathered}
e^{-}+\mathrm{H}_{2} \rightarrow \mathrm{H}_{2}^{+}+2 e^{-}, \\
e^{-}+\mathrm{H}_{2} \rightarrow 2 \mathrm{H}+e^{-} .
\end{gathered}
$$

The energetic photoelectrons quickly lose energy by collisions with the neutral hydrogen molecules and resulting secondary ionizations, dissociations, and excitations or are lost to the walls. As shown in Fig. 9, the dominant electron-impact process is ionization to $\mathrm{H}_{2}{ }^{+}$, with a branching ratio of $\sim 60 \%$ for $76 \mathrm{eV}$ electrons, and on average $\sim 0.6$ additional electrons are formed per collision. The ionization energy loss is $15.4 \mathrm{eV}$, and the remaining energy is divided over the electrons, or an (average) electron energy of $\sim 30 \mathrm{eV}$. This may still be sufficient for an additional ionization and dissociation, after which the electron energy will have dropped below $10 \mathrm{eV}$ and further collisions will only result in excitations of electronic and ro-vibrational states. Combining these ionizing and dissociating steps results in a potential formation of $\sim 2.5$ ions per absorbed photon and up to eight hydrogen radicals. For a confined plasma, a finite fraction of the electrons is lost to the wall, and the number of ions and radicals generated is lower.

The inelastic mean-free path $\lambda_{e}$ and collision frequency $f_{\text {en }}$ of the electrons are (neglecting the inefficient energy transfer by momentum transfer for electron energies above $1 \mathrm{eV}):{ }^{34}$

$$
\begin{gathered}
\lambda_{e}(\varepsilon)=\frac{1}{\sigma_{\mathrm{en}}(\varepsilon) \cdot n_{\mathrm{H} 2}}, \\
f_{\mathrm{en}}(\varepsilon)=\frac{v_{e}(\varepsilon)}{\lambda_{e}(\varepsilon)}=\sqrt{2 \varepsilon / m_{e}} \cdot \sigma_{\mathrm{en}}(\varepsilon) \cdot n_{\mathrm{H} 2},
\end{gathered}
$$

where $\varepsilon$ is the electron energy, $v_{e}$ is the electron velocity, and $\sigma_{\mathrm{en}}$ is the energy-dependent electron-neutral cross section for inelastic collisions. According to Yoon, the cumulative inelastic cross section for electron energies of $>25 \mathrm{eV}$ is $\sigma_{\mathrm{en}}=1.7 \cdot 10^{-20} \mathrm{~m}^{2}$ at $5 \mathrm{~Pa} \mathrm{H}_{2},{ }^{32}$ resulting in an initial mean free path of $\lambda_{e}=4.9 \mathrm{~cm}$ and collision frequency $f_{\text {en }}$ of $\sim 100 \mathrm{MHz}$. For an EUV beam width of $\sim 1 \mathrm{~cm}$ or smaller, this means that the majority of the fast electrons escape the beam volume and secondary ionizations and radical formation mainly takes place outside the original beam volume. In addition, the high initial electron energy leads to a rapid initial 
expansion of the plasma to fill the surrounding $\sim 1 \mathrm{~cm}$ around the beam in the first $\sim 0.1 \mu \mathrm{s}$, after which the expansion rate drops as the electrons cool down.

Ion-molecule collisions of the cold $\mathrm{H}_{2}^{+}$ions with $\mathrm{H}_{2}$ result in $\mathrm{H}_{3}^{+}$by the highly efficient proton hop mechanism, resulting in $\mathrm{H}_{3}^{+}$as the dominant ion after $\sim 0.5 \mu \mathrm{s}$, as also observed in the interstellar hydrogen plasma. ${ }^{35}$ The $\sim 1.7 \mathrm{eV}$ energy released in this exothermic process is distributed between internal excitation of the molecular ion and kinetic energy of the $\mathrm{H}$ radical and the molecular ion, ${ }^{33}$ which again is a source of relatively hot radicals:

$$
\mathrm{H}_{2}^{+}+\mathrm{H}_{2} \rightarrow \mathrm{H}_{3}^{+}+\mathrm{H}
$$

The interactions of $\mathrm{H}^{+}$with $\mathrm{H}_{2}$ are dominated by momentum transfer, although asymmetric charge transfer to $\mathrm{H}_{2}^{+}$is also possible, e.g., when the $\mathrm{H}^{+}$ions are accelerated in the sheath to $>3 \mathrm{eV}\left(\sigma=\sim 10^{-20} \mathrm{~m}^{2}\right) .{ }^{36}$ The resulting ion distribution after the first microsecond is roughly $90 \% \mathrm{H}_{3}^{+}$and $10 \% \mathrm{H}^{+}$.

\subsubsection{Photoelectric effect}

Mirrors and construction materials emit electrons when irradiated by photons with an energy $h \nu$ above the work function of the material. ${ }^{37}$ The quantum efficiency is governed by the penetration depth of the photons (which increases with photon energy) and hence in general decreases for higher photon energy. ${ }^{38}$ In terms of irradiance (\#e/W), this is further exacerbated by the lower number of photons per joule for higher photon energy. For metals, the electron quantum yield is typically in the order of a few percent for EUV photons. For the ruthenium $(\mathrm{Ru})$ mirror cap layer, the secondary electron yield (SEY) has been measured to be $2 \%$, with an electron energy spectrum dominated by low-energy electrons, ${ }^{39}$ which may reasonably be approximated by a Maxwellian distribution with $T_{e} \approx 3 \mathrm{eV} .{ }^{40}$

For the example of the RME, conservatively estimating the reticle SEY to be $2 \%$ and taking a photon flux of $10^{21} \mathrm{~s}^{-1} \cdot \mathrm{m}^{-2}$, the secondary electron current density may be estimated to be $\sim 2 \cdot 10^{19} \mathrm{~s}^{-1} \cdot \mathrm{m}^{-2}$ (or $\sim 3 \mathrm{~A} / \mathrm{m}^{2}$ ), which is larger than the gas ionization contribution. In practice, this current is be self-limiting by formation of an instantaneous negative space charge layer next to the surface that traps the low-energy electrons and returns these to the surface. In combination with simultaneous gas ionization by EUV, this negative space layer is partially compensated for by the overlap with the positive space layer of the ionized gas some electrons, allowing more low-energy secondary electrons to escape from the surface and more high-energy photoelectrons to escape to the surface. The electron flux from the surface during the EUV pulse suppresses formation of a classical sheath during the pulse, and the plasma-to-surface potential is reduced in this phase. At the end of EUV pulse, the photoelectric electron flux stops, and a classical sheath develops, but with a delay and reduced maximum potential. ${ }^{30}$ This significantly reduces the transient electron energies and resulting peak ion energies.

The hydrogen plasma is significantly modified by the relatively cold electrons from the photoelectric effect in the vicinity of the reticle or a mirror or other EUV-irradiated surface. The secondary electrons form a significant population with respect to the energetic electron from gas ionization, and the resulting plasma must be treated as a bi-Maxwellian distribution. This significantly reduces the transient electron energies and resulting plasma characteristics.

As the work function of most metals and construction materials is in the order of $5 \mathrm{eV}$, all outof-band radiation with wavelengths up to $200 \mathrm{~nm}$ should be considered for a total photoelectric effect. During the EUV pulse, the out-of-band contribution of $\sim 1 \%$ may be ignored as a minor perturbation, but these wavelengths may persist in the cooling-down phase after the EUV pulse as a UV afterglow, which may continue to release low-energy photoelectrons for several tenths of microseconds after the EUV has died out (see Sec. 2). These UV photons do not ionize but do generate additional low-energy secondary photoelectrons from irradiated surfaces, thereby charging the plasma volume negatively for a longer time.

Even a minor UV afterglow of $\sim 1 \%$ can have a significant effect on the plasma, since both the quantum efficiency and the number of photons per watt is roughly an order of magnitude higher for UV than for EUV. ${ }^{37}$ Thus, the number of photoelectrons generated in the afterglow may be in 
same order of magnitude as the EUV-induced plasma electrons, and the development of a classical sheath is delayed until the afterglow dies out.

\subsubsection{Wall losses, electron exchange, and electron cooling}

For a closely confined plasma, loss of energetic electrons to the walls may dominate over inelastic or ionizing collisions, with a fraction $f_{w}$ of the energetic photoelectrons being lost to the wall without further ionizing collisions events:

$$
f_{w}=e^{-\frac{\Lambda_{w}}{\lambda_{e}}}
$$

where $\Lambda_{w}$ is the typical length of the confinement and $\lambda_{e}$ is the mean-free path of the electrons. For a confined EUV-induced plasma with $\Lambda_{w}=1.5 \mathrm{~cm}$ and $\lambda_{e}=4.9 \mathrm{~cm}$, a significant fraction of $f_{w} \approx 74 \%$ of the absorbed EUV energy may be lost to the walls and significantly less secondary ionizations take place, resulting in a $\sim 2 \times$ lower plasma density as compared with an open plasma. $^{17}$

Previous investigations assumed that this would be counteracted by the almost instantaneous formation of a potential well that subsequently repels and confines the majority of fast electrons. ${ }^{17,41}$ However, for the high-energy photoelectrons from EUV ionization, SEE from the surface becomes a significant effect, which so far seems to have been overlooked. For typical construction materials, such as stainless steel and aluminum, the yield of (low-energy) secondary electrons has been measured to be above unity for $76 \mathrm{eV} .{ }^{42}$ In other words, the plasma-facing surfaces tends to charge positive rather than negative, so no potential well is formed near walls and no electron trapping or ion acceleration occurs in this phase.

This SEY $>1$ for $76 \mathrm{eV}$ is not generally predicted by the universal curve, ${ }^{43}$ which was set up with a focus on higher energy electrons and ideal metal surface states. For energies well below the maximum yield energy (typically 0.4 to $0.6 \mathrm{keV}$ for construction materials), it is advisable to use an empirical linearization based on literature data and/or measurements and to blend that smoothly into the universal curve above $\sim 100 \mathrm{eV}$. This is the approach that was incorporated into our 3D-PIC model.

The secondary electrons have lower energies and may be approximated by a Maxwellian distribution with $T_{e} \approx 3 \mathrm{eV}$, similar to for photogenerated secondary electrons. The resulting overall electron distribution is bi-Maxwellian with a split in low-energy and high-energy fractions and an average energy well below $10 \mathrm{eV}$. As the remaining plasma electrons cool down by collisions to below $30 \mathrm{eV}$ after $25 \mathrm{~ns}$, the SEY drops below unity, and the wall starts to charge negatively. A classical plasma-wall sheath subsequently develops, albeit at a later time and with significantly lower sheath potential than if no SEE is taken into consideration. The details of this transition depend strongly on wall material and surface finishing, as illustrated in Fig. 10 for the example of chrome $(\mathrm{Cr})$, and no generic analytical solution can be given.
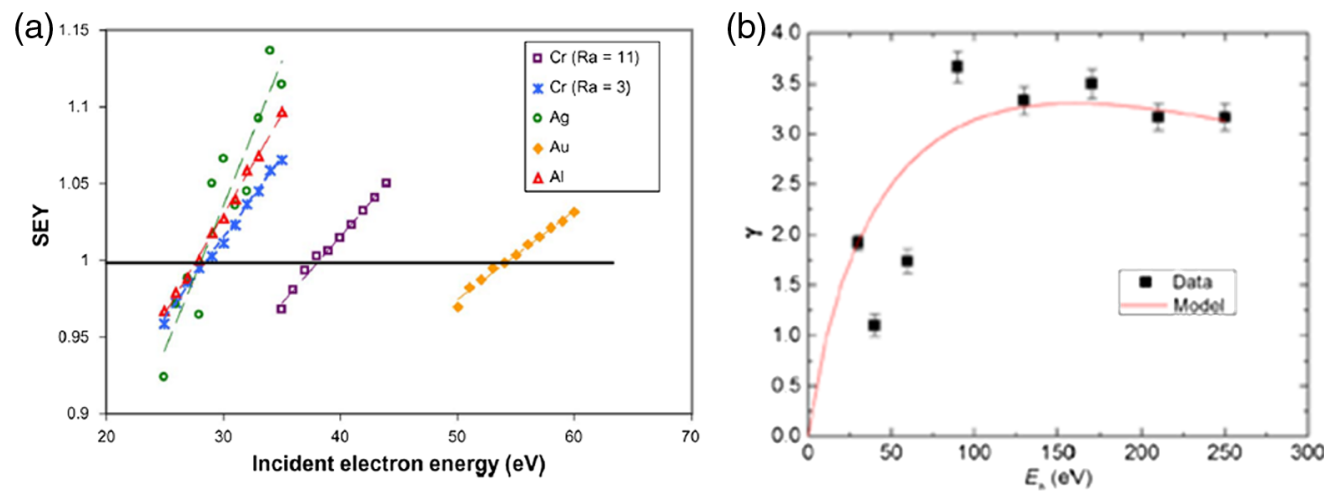

Fig. 10 Examples of SEY as function of incident electron energy. (a) $\mathrm{Cr}$ (with different levels of roughness $R_{a}=3$ and $11 \mu \mathrm{m}$ ), Al, Ag, Au; from Ref 41. (b) stainless steel; from Ref. 44. 


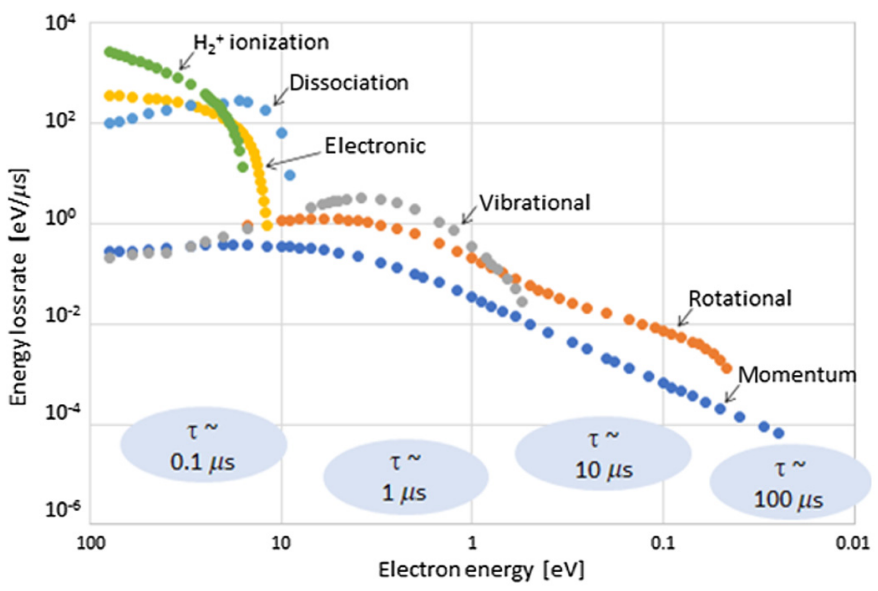

Fig. 11 Electron energy loss rates by inelastic collisions; based on data from Refs. 34 and 32.

In addition to electron exchange at the walls, the energetic photoelectrons lose their energy fast in ionizing and dissociating collisions with the neutral molecules. The initial electron energy distributions from photoionization is not in thermal equilibrium and does not follow a Maxwellian distribution; this is significant for the resulting plasma properties until the electrons cool down to below the ionization threshold, after which the plasma may be satisfactorily approximated by a Maxwellian distribution. As the electron energy drops below $10 \mathrm{eV}$, further cooling proceeds at a progressively lower rate by ro-vibrational excitations and finally momentum transfers. Thermalization by electron-electron collisions becomes relevant only when the plasma has cooled down to below $1 \mathrm{eV} .^{17}$

The electron energy loss rate changes stepwise as different collision types with different energy losses become less or more important; also, the gradual decrease in collision frequency with decreasing energy results in a gradual reduction of cooling rate, as shown in Fig. 11. The initial electron energy of $75 \mathrm{eV}$ drops to below $10 \mathrm{eV}$ within $0.1 \mu \mathrm{s}$ and to below $0.5 \mathrm{eV}$ within $5 \mu \mathrm{s}$. Further cooling and thermalization by elastic collisions are slow and may take $>100 \mu \mathrm{s}$. The energy decrease over time may be approximated by piecewise exponential functions with increasing time constants for the different energy ranges.

It should be noted that, as the density of metastable vibrationally excited $\mathrm{H}_{2}$ molecules builds up, an equilibrium in which electrons also experience superelastic collisions might form, and a long-lasting electron fraction with $0.5 \mathrm{eV}$ energy may be sustained (corresponding to the vibrational energy quantum of $\left.0.516 \mathrm{eV}^{45,46}\right)$.

\subsubsection{Diffusion, flux, and energy}

In the plasma bulk, where there is no electrical field, the velocity of the electrons depends on their energy, $v_{e}=\sqrt{2 \varepsilon / m_{e}}$, and may be as high as $\sim 10^{6} \mathrm{~m} / \mathrm{s}$. As the electron energy drops to $1 \mathrm{eV}$, the velocity becomes in the order of $10^{5} \mathrm{~m} / \mathrm{s}$. Though very high, such an electron speed is finite with respect to the fast transients, and for a typical distance of $1 \mathrm{~cm}$ may result in finite delays in the order of $0.1 \mu \mathrm{s}$.

At the edge of the plasma, quasineutrality is no longer valid, and the electrons are decelerated by the coulomb interaction with the heavier and slower ions; this mutual interaction results in ambipolar diffusion, slowing down the electrons and speeding up the ions. During the EUV pulse, for a confined plasma, the entire volume is filled with ions via primary and secondary ionizations, after which the ions diffuse out toward the walls. The ambipolar diffusion constant $D_{a}$ scales with pressure and electron temperature: ${ }^{47}$

$$
D_{a}=\mu_{i}^{0} \cdot \frac{p_{0}}{p_{\mathrm{H} 2}} \cdot\left(T_{e}+T_{i}\right) \cong \mu_{i}^{0} \cdot \frac{p_{0}}{p_{\mathrm{H} 2}} \cdot T_{e} .
$$


With $\mu_{i}^{0}=1.1 \cdot 10^{-3} \mathrm{~m}^{2} / \mathrm{Vs}$ the ion mobility of $\mathrm{H}_{3}^{+}$in hydrogen at atmospheric pressure $\left(p_{0}=10^{5} \mathrm{~Pa}\right),{ }^{48} p_{\mathrm{H} 2}$ is the hydrogen pressure in $\mathrm{Pa}$, and $T_{e, i}$ is the electron and ion temperatures in $\mathrm{eV}$. The continuum assumption underlying Eq. (18) is only a rough approximation for a biMaxwellian distribution, and more rigorous numerical PIC-models are needed for the transient phase during and after the EUV pulse.

In the case of a bi-Maxwellian split electron energy distribution, the effective electron temperature may be a function of distance to the wall, which in turn may give rise to discontinuities in the diffusion-driven ion flux for different time-scales, with the short-term being driven by the high-energy fraction $\left(T_{e 2}\right)$ and the long-term by the average electron temperature $\left(T_{e 1}\right)$.

As the plasma ions diffuse outward and reach the surrounding walls, they recombine with the electrons already present at the wall surfaces, and the lifetime of the plasma pulse is thus limited by ambipolar diffusion. In view of the low-ionization degree, volume recombination is a minor effect; whether it can be ignored completely depends mainly on pressure and wall distance. ${ }^{49}$ The ambipolar diffusion time constant is

$$
\tau_{a}=\frac{\Lambda_{w}^{2}}{D_{a}} \cong \frac{\Lambda_{w}^{2} \cdot p_{\mathrm{H} 2}}{\mu_{i}^{0} \cdot p_{0}} \cdot \frac{1}{T_{e}},
$$

where $\Lambda_{w}$ is the typical length of the plasma confinement, which for a rectangular geometry may be found from

$$
1 / \Lambda_{w}^{2}=1 / L_{x}^{2}+1 / L_{y}^{2}+1 / L_{z}^{2}
$$

The RME may be approximated by a rectangular box with $L_{x}=12 \mathrm{~cm}, L_{y}=2 \mathrm{~cm}$ (the beam itself is $\sim 11 \times 1 \mathrm{~cm}^{2}$, as described in Sec. 2; the $z$-dimension of the box is arbitrary and may be taken as equal to the smallest dimension, or $L_{z}=2 \mathrm{~cm}$ ). This results in a typical length of $\Lambda_{w} \approx 1.5 \mathrm{~cm}$ for the RME. Taking $\Lambda_{w}=1.5 \mathrm{~cm}$ and an electron temperature of $0.1 \mathrm{eV}$, a typical value for the long-term diffusion time constant is $\tau_{a} \cong 100 \mu \mathrm{s}$. It should be noted that diffusion losses are slower for higher pressure, whereas the collisional losses are faster for higher pressure.

When the sheath has formed at the plasma-facing surface, all ions entering the sheath from the presheath are accelerated toward the surface. The ion flux to the wall $\varphi_{i, w}$ is the product of the ion density in the presheath $n_{i, \mathrm{ps}}$ and the Bohm velocity. The ion density in the presheath is determined by the ambipolar flow velocity from the EUV beam toward the surface. Diffusional transport takes a relatively long time from beam to wall, so it does not respond to the instantaneous value of the electron temperature. The Bohm velocity on the other hand is proportional to the instantaneous value of $\sqrt{T_{e 2}}$, and this drives the dynamics of the ion flux to peak with the transient peak and rapid fall in $T_{e 2}$ during and directly after the EUV pulse.

As outlined above, the initial sheath formation at the plasma-facing surfaces is frustrated by SEE. In the initial phase with SEY > 1, a space-charge-limited negative sheath layer (SCL) ${ }^{50}$ or even an inverse sheath, ${ }^{51}$ forms near the walls. As the photoelectrons cool down to below $\sim 30 \mathrm{eV}$, the SEY drops below unity. As shown in Fig. 12(a), the SCL sheath then transitions to a classical plasma-wall sheath, albeit with reduced sheath potential. As the classical sheath is formed, the ion flux starts to rise on a timescale of a fraction of a microsecond, limited by ion inertia and the finite force of the electrical sheath field. The delay in the formation of a classical sheath results in a dead period in ion flux after the EUV pulse; also by the time the sheath is formed, the electrons have cooled down to energies below $10 \mathrm{eV}$, and the effective electron temperature is in the order of $\sim 1 \mathrm{eV}$.

The SCL period may be extended by the UV afterglow of the EUV pulse, which for an LPP source may continue to release low-energy photoelectrons for up to $\sim 0.3 \mu$ s after the EUV has died out, maintaining a negative space-charge layer during that time (see Sec. 2).

As the classical sheath is formed over a time span of $\sim 0.1$ to $0.2 \mu$ s, the ion energy rises with the increasing plasma-wall potential, as well as the ion flux. However, simultaneously the overall electron energies are decreasing fast, as is correspondingly both sheath potential and Bohm velocity. The combined effect is a sharp peak followed by an exponential decrease in both ion energy and ion flux, following the decrease in fast electron energy, as shown in Fig. 12(b). 
(a)

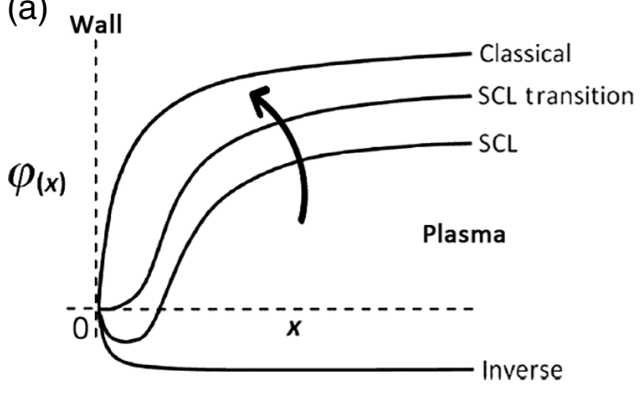

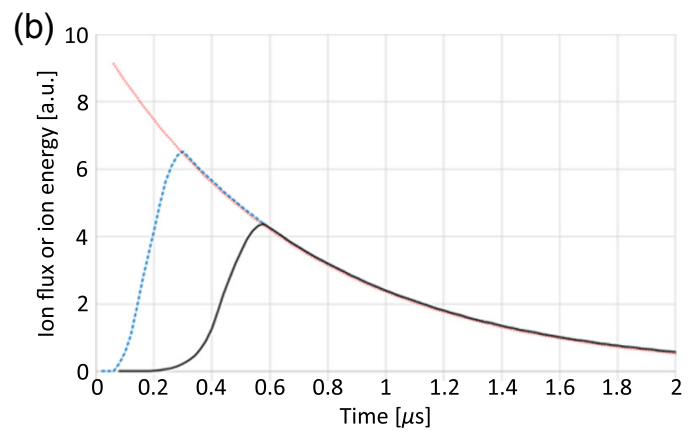

Fig. 12 (a) Sheath evolution over time, including SEE from fast electrons and UV afterglow; adapted from Ref. 50. (b) Artist impression of the ion flux/energy transient by the intersection of sheath formation and electron cooling curve (dotted red line), without (dashed blue line), and with delay from SCL (solid black line).

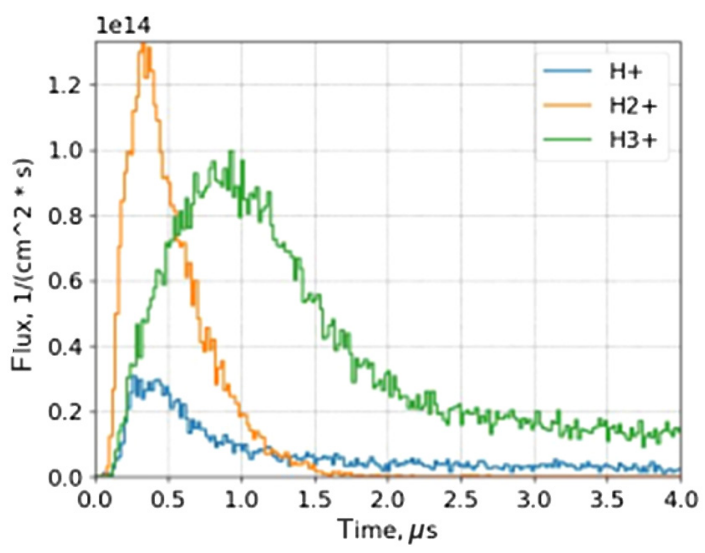

Fig. 13 Modeled transient ion dynamics at the edge of the EUV beam (beam width $1 \mathrm{~cm}$ ).

These phases of dead time, peak, and decrease also show clearly in the PIC model results for both ion flux and ion energy, as shown in Fig. 13. For hydrogen, the transient period in the first microsecond is convoluted with the overlapping transition from $\mathrm{H}_{2}^{+}$to $\mathrm{H}_{3}^{+}$, which leads to the later peak in $\mathrm{H}_{3}^{+}$and results in $\mathrm{H}_{2}^{+}$as the most abundant energetic species, even if $\mathrm{H}_{3}^{+}$is the most abundant ion overall. Detailed interpretation of hydrogen ion spectra requires an approach that is selective to ion species as well as time-resolved.

\subsubsection{Volume recombination}

Although earlier work reported that volume recombination could be ignored at pressures of $10 \mathrm{~Pa}$ and below, internal measurements have shown that this is not always the case. For volume recombination, both binary and ternary recombination should be considered, with reaction rates of $k_{\mathrm{bin}}=\sim 2 \cdot 10^{-8} \mathrm{~cm}^{3} \mathrm{~s}^{-1}$ (for $\sim 0.1 \mathrm{eV}$ electrons) and $k_{\mathrm{tern}}=8.7 \cdot 10^{-23} \mathrm{~cm}^{6} \mathrm{~s}^{-1}$, respectively. ${ }^{52,53}$

$$
\begin{gathered}
\mathrm{H}_{3}^{+}+e^{-\stackrel{k_{\text {bin }}}{\rightarrow}} \mathrm{H}_{2}+\mathrm{H}, \\
\mathrm{H}_{3}^{+}+\mathrm{H}_{2}+e^{-\stackrel{k_{\text {tem }}}{\longrightarrow}} 2 \mathrm{H}_{2}+\mathrm{H} .
\end{gathered}
$$

Close to room temperature, the ternary recombination is expected to dominate over the binary dissociative recombination for hydrogen pressures above $\sim 2 \mathrm{~Pa}\left(n_{\mathrm{H}_{2}} \cong 5 \cdot 10^{20} \mathrm{~m}^{-3}\right.$, and $n_{\mathrm{H}_{2}} \cdot k_{\text {tern }}>k_{\text {bin }}$ ). Ion generation scales with EUV with pressure (and intensity), and diffusion 


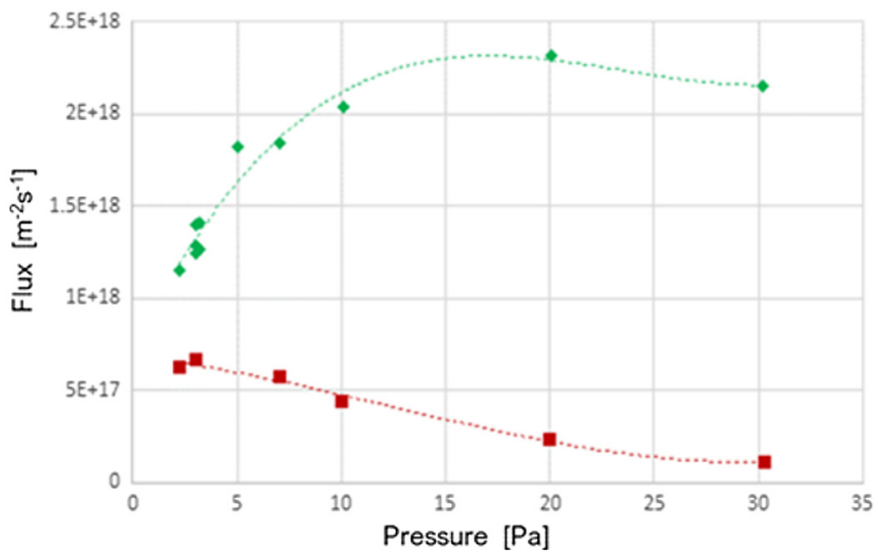

Fig. 14 Ion flux as function of pressure, measured at the edge of the EUV beam (diamonds) and at a distance of $4 \mathrm{~cm}$ (squares) from the beam edge. ${ }^{54}$

transport scales with pressure (and inversely with distance to the wall), whereas ternary recombination scales with pressure to the third power (and with distance). For very low pressures, the ion flux increases with pressure at any given distance. For higher pressures, the third-power pressure scaling of recombination results in a balance of ion source and loss terms, and the ion flux reaches a maximum; beyond that the ion flux decreases for a further pressure increase. Recombination and diffusion also depend on distance, shifting the location of this maximum to lower pressures further away from the beam. For a given distance, increasing EUV power shifts the maximum to a somewhat lower pressure.

For $5 \mathrm{~Pa}$, and confinement with typical length $\Lambda_{w}=1.5 \mathrm{~cm}$, Fig. 14 shows that the recombination is minor and ion flux to the walls scales more or less linearly with pressure and with power. For a less confined, more open plasma, this transition point shifts to a lower pressure, in which case recombination may become significant also for $5 \mathrm{~Pa}$.

\subsubsection{Pulsed mode operation}

The LPP EUV source operates at a cycle time of $20 \mu$ s, with an EUV pulse length of $<100 \mathrm{~ns}$. As the plasma does not extinguish completely within $20 \mu$ s, there is a pulse-by-pulse build-up toward a quasisteady-state plasma, with repeating transient peaks every $20 \mu \mathrm{s}$. During scanner exposures, the EUV pulse train typically runs for $\sim 5000$ pulses and is then interrupted for several milliseconds, during which time the plasma extinguishes completely. This mode of operation means that the vast majority of pulses are fired on top of a steady-state background plasma and start-up and decay effects may effectively be ignored.

Extending the single-pulse bi-Maxwellian treatment above, there will initially be three electron populations in the transient phase after the pulse: a hot fraction driven by gas ionization, a cooler fraction driven by SEE from the walls, and a cold fraction from the background plasma. In this case, the gas ionization fraction, with the highest energy, defines $T_{e 2}$ initially, and the collisional cooling of these electrons drives the plasma potential transient after the pulse. However, as outlined above, the negative space charge from the secondary electrons from the wall might prevent a classical sheath build-up in the first $\sim 0.2$ to $0.3 \mu$ s, so this might not translate one on one into ion energies. After $\sim 2 \mu \mathrm{s}$, the populations effectively merges, and the afterglow and global diffusion are driven by the average electron temperature.

For an open plasma, ambipolar diffusion is much slower than the pulse cycle and plasma builds up until volume recombination balances plasma generation at high enough electron and ion densities; the pulses can then be treated as perturbations and the plasma can be reasonably approximated by a continuous ionization source of same average power instead of a pulsed plasma, enabling continuum theories and corresponding fluid models. ${ }^{55}$ For a confined plasma, or very close to the mirror or reticle, the diffusion losses to the surface limit the build-up and the plasma properties may to a large degree be determined by the repeating transient peaks; this requires explicit PIC modeling and nonlocal kinetics. ${ }^{18}$ 


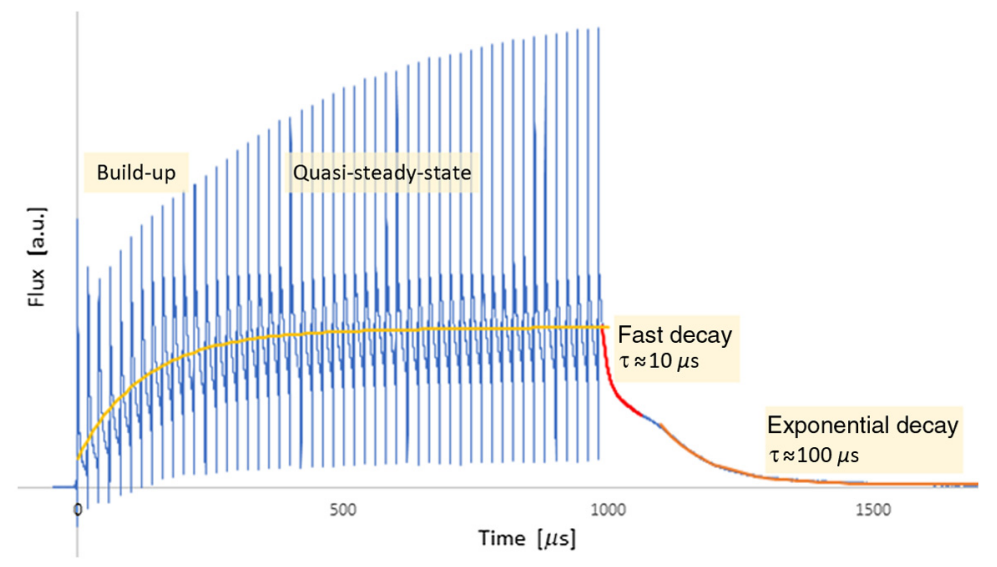

Fig. 15 Build-up, steady-state, and decay of pulsed EUV-induced plasma, as measured by RFEA in LPP testrig at $5 \mathrm{~Pa}^{53}$

For a relatively open plasma, Fig. 15 shows the measured buildup and decay of the ion flux and the pulses on top of an evolving background. Both the global build-up and long-term decay can be fitted by an exponential diffusion with time constant in the order of $\tau_{a} \cong 100 \mu \mathrm{s}$, which corresponds to a background electron temperature close to room temperature. As may be observed, the decay for the first $\sim 50 \mu$ s after the last EUV pulse, and in between the EUV pulses, is significantly faster with a time constant of $\sim 10 \mu \mathrm{s}$, corresponding to a significantly higher apparent $T_{e} \cong 0.5 \mathrm{eV}$. This may be qualitatively explained by a bi-Maxwellian split electron population in which part of the electrons receive energy from superelastic collisions with vibrationally excited hydrogen molecules, with a vibrational energy quantum of $0.516 \mathrm{eV} ;{ }^{17}$ similar behavior has been observed also in other pulsed hydrogen ${ }^{56}$ and $\operatorname{argon}^{57}$ plasmas. The flux decay rate in this phase is driven by the cooling rate of the high-energy electron fraction $\left(T_{e 2}\right)$, which is driven by collisions and thus scales with pressure $\left(\tau_{a \text {,ini }} \sim 1 / p\right.$; whereas the long-term diffusiondriven decay rate scales inversely with pressure $\tau_{a} \sim p$ ).

The peaks in ion flux and energy occur when the sheath formation intersects with the photoelectron cooling curve. This intersection depends on wall geometry, pressure, and background plasma. The exponential energy decay is driven by collisional cooling, with $f_{\text {en }}$ scaling linearly with pressure, and does not depend on distance. This faster cooling results in a lower ion energy by the time the sheath is formed. However, this is (partly) balanced by faster sheath formation for higher pressure; as for higher pressure, the conductivity of the plasma increases and the initial negative space charge layer from the SEE term is dissipated faster. The net result is that peak energy drops with increasing pressure, as shown in Fig. 16(b).

(a)

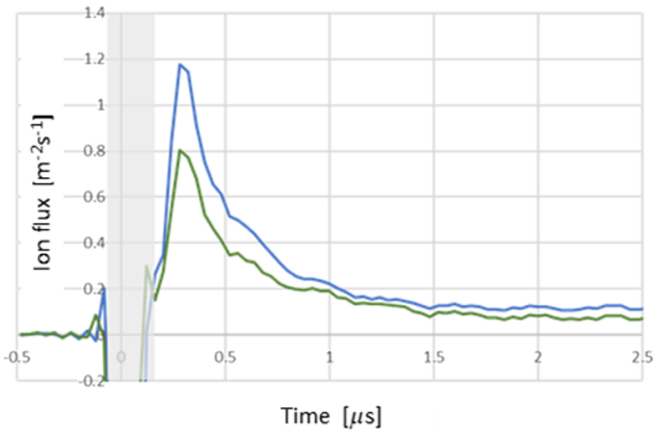

(b)

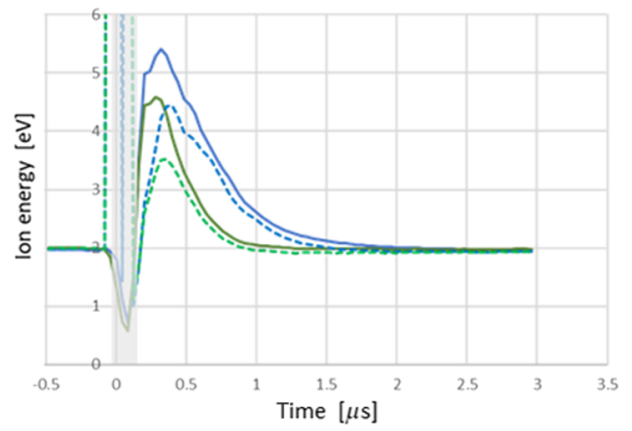

Fig. 16 RFEA measurement of peak ion energy and flux in quasi-steady-state; RFEA blind time of $0.2 \mu \mathrm{s}$ is marked in gray. (a) lon flux peak measured at $4 \mathrm{~cm}$ from the beam edge. (b) lon energy peak for pressure of 5 (blue) and $10 \mathrm{~Pa}$ (green), at $0 \mathrm{~cm}$ (solid lines) and at $4 \mathrm{~cm}$ (dashed lines) from the EUV beam edge. 
In between the pulses, the ion energy drops fast to a metastable platform of roughly $2 \mathrm{eV}$, which corresponds to an electron temperature $T_{e} \cong 0.5 \mathrm{eV}$. This is significantly higher than the expected $T_{e}<0.1 \mathrm{eV}^{17}$ but is consistent with the measured decay time constant, as well as with earlier off-line experiments. ${ }^{45}$ As discussed above, this may be explained by superelastic collisions with vibrationally excited hydrogen molecules.

Figure 16 also shows that at $4 \mathrm{~cm}$ distance from the beam, the ion peak is delayed a further $80 \mathrm{~ns}$ and the peak energy is accordingly lower, intersecting with the cooling curve at a later time; this may be explained by the finite speed of the electrons traveling between the EUV beam and the surface/sensor $\left(v_{e} \cong 6 \cdot 10^{5} \mathrm{~m} / \mathrm{s}\right.$ around $\left.1 \mathrm{eV}\right)$, resulting in delayed formation of plasma potential and sheath at larger distances. At $4 \mathrm{~cm}$ distance from the beam, the ion flux is observed to decrease for higher pressure, driven by faster cooling, and resulting in slower diffusion to this distance, consistent with Fig. 14. Perhaps surprisingly, a higher pressure may thus result in a reduced ion load to plasma-facing surfaces, both in terms of flux and peak energy (for a wall sufficiently far from the EUV beam). This could be considered an artifact of a relatively open plasma; for a more closely confined plasma with $\Lambda_{w}=1.5 \mathrm{~cm}$, ion flux increases for higher pressure until $>10 \mathrm{~Pa}$.

As diffusion is relatively slow, the fast exponential decay of the flux is mainly driven by the evolution of the Bohm velocity. However, the sheath width may change fast, in which case less or more ions are captured by the sheath; thus, the flux decay curve over time might be more complex and might show dips (when sheath decreases) or bumps (when sheath increases). The coinciding peaks in ion energy and flux result in an enhanced fraction of high-energy ions, which may be significant for the plasma-wall interactions, as these typically exhibit nonlinear response to ion energy, such as sharp energy thresholds, e.g., sputtering (when exceeding the sputtering threshold) or ion-enhanced chemical reactions (when exceeding the surface binding energy).

During the first pulses of the burst, the background plasma contribution grows, again reducing the relative contribution from the SEE terms and thus allowing the sheath to form faster; this results in increasing ion energy peak, as shown in Fig. 17(a). The balance between the different electron contributions from gas ionization and SEE terms is also influenced by the source frequency, with a longer pulse interval resulting in a lower background plasma at the next pulse. This increases the relative contribution from the SEE terms and delays the sheath formation, resulting in a lower ion energy peak for longer pulse interval, as shown in Fig. 17(b). Conversely, increasing the source frequency reduces the pulse interval and results in higher ion peak ion energies.

Despite the qualitative understanding of the underlying mechanisms, the shifting balance between gas ionization and SEE terms cannot be described analytically, and (hybrid) PIC modeling is required. However, a PIC model also relies on many manual input parameters, e.g., the duration of the UV afterglow and the actual SEE of real materials and surfaces, so model validation remains crucial for each EUV source type and for each vacuum vessel design.
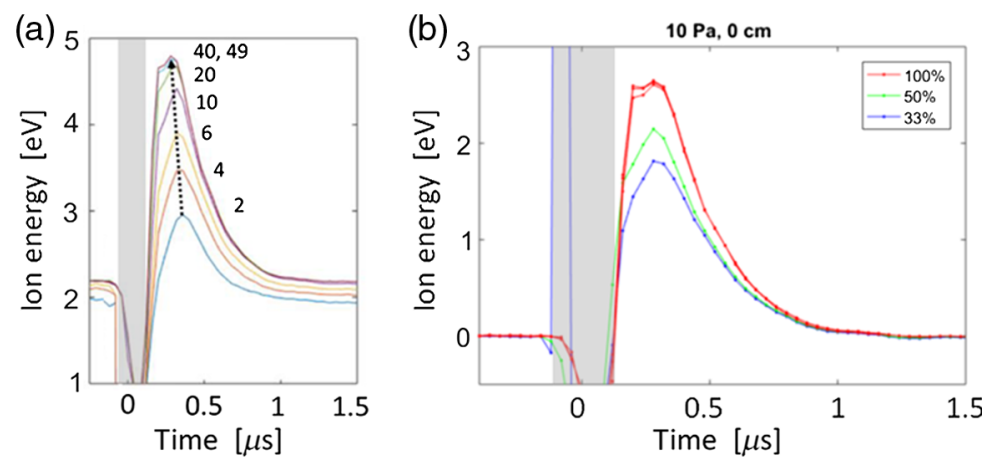

Fig. 17 RFEA measurement of peak ion energy, at $10 \mathrm{~Pa}$, next to the EUV beam. (a) lon energy as function of pulse number at start of the burst. (b) lon energy as function of pulse duty cycle $(100 \%=20 \mu \mathrm{s}$ pulse interval; $50 \%=40 \mu \mathrm{s}, 33 \%=60 \mu \mathrm{s})$. RFEA blind time of $0.2 \mu \mathrm{s}$ is marked in gray. 
The extended tail with $2 \mathrm{eV}$ is not predicted by the model, most likely because the superelastic effect is not captured sufficiently in the model. There is also some discussion on the absolute value of the RFEA energy measurements. During the EUV pulse, the RFEA is clearly affected by spurious photoelectric effects (grayed-out zones in Figs. 16 and 17). Also, especially in the fast transient after the EUV pulse, some crosstalk between readout and grids cannot be excluded. Work has started to install an EQP on the LPP source testrig to verify these aspects of the RFEA.

\subsubsection{Radicals}

As outlined above, neutral $\mathrm{H}^{*}$ radicals are formed in and near the EUV beam and diffuse out to the walls. However, the radicals only have a finite likelihood of wall recombination, and for a confined plasma the radical density builds up much more than the ions. The radical recombination coefficient $\gamma$ of the walls may vary significantly for different materials and is also significantly influenced by the surface state; for clean construction metals, it may be assumed to be in the order of $0.1{ }^{58}$ Furthermore, wall recombination of the $\mathrm{H}_{3}^{+}$ions creates $\sim 2 \mathrm{H}$-radicals per recombining ion on average..$^{59}$ Volume recombination of radicals requires three-body collisions in view of momentum and energy conservation, so it may typically be ignored.

This combination of finite recombination probability and generation at the walls leads to significant build-up of radical flux. One of the important parameters in plasma chemistry is the flux ratio of radicals to ions, which is governed by the respective photon yields and the radical recombination coefficient (taking the ion recombination coefficient to be unity and approximating ion and radical motion by uniform diffusion):

$$
\frac{\varphi_{r}}{\varphi_{i}} \cong \frac{1}{\gamma} \cdot \frac{\eta_{r}}{\eta_{i}}
$$

where $\varphi_{r}$ and $\varphi_{i}$ are the radical and ion fluxes and $\eta_{r}$ and $\eta_{i}$ are the respective yields per photon. Working out the different reaction pathways for ionization, dissociation, and recombination, the yield ratio is roughly five radicals per ion. Assuming a recombination coefficient $\gamma=0.1$, radical fluxes are $\sim 50 \times$ higher than ion fluxes. It should be noted that, in contrast to ions, the resulting radical density distribution is more or less constant in the volume around the EUV beam and does not show appreciable peaking in the beam itself, so the radical-to-ion ratio is lower inside the beam (and near the mirror surface) and higher further away from the beam. A further complicating factor may be that the detailed distribution of ion flux over the different surfaces may be affected by the details of the respective sheath fields.

The radicals do not respond to the fast transient electron energy peaks and may be treated as a continuous flux.

The production processes of radicals result in high kinetic energies of the radicals of roughly 1 to $8 \mathrm{eV}$ (for dissociative ionization), which are thermalized by momentum exchange with the background gas and the walls within a few $\mu$ s. The majority of the radical flux may thus be assumed to be close to room temperature, but with a broad distribution and a tail with high energies. These high-energy radicals may be significant for the finite activation energies in surface reactions and for overcoming absorption barriers of hydrogen into metals to increase the absorbed hydrogen concentration.

\subsubsection{Gas flow}

For completeness, the loss term by flow (or convection) also should be considered, as gas in the scanner or test setup is typically circulated. This is given by the convection residence time $\tau_{\mathrm{CR}}$ :

$$
\tau_{\mathrm{CR}}=\frac{\Lambda_{p}}{v_{\mathrm{H} 2}},
$$

where $\Lambda_{p}$ is the plasma dimension in the flow direction and $v_{\mathrm{H} 2}$ is the flow velocity. For a typical flow of $v_{\mathrm{H} 2}=100 \mathrm{~m} / \mathrm{s}$ and a plasma confinement size of $\Lambda_{p}=2 \mathrm{~cm}, \tau_{\mathrm{CR}} \cong 0.2 \mathrm{~ms}$, which is 

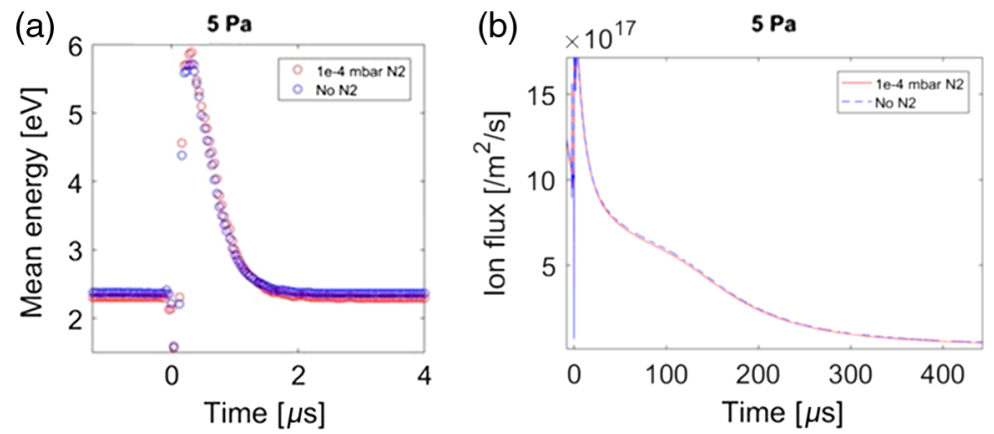

Fig. 18 Transient ion energy after EUV pulse, with and without addition of $\mathrm{N}_{2}$, measured in LPP testrig at the edge to the beam for $5 \mathrm{~Pa}$. (a) Fast transient and (b) long-term decay after last pulse. $^{53}$

higher than the ambipolar diffusion constant above but not by orders of magnitude. The flow may therefore have a modest impact, increasing the effective decay rate by $\sim 10 \%$. As this effect is small with respect to the other uncertainties, gas flow has for now been excluded from our 3D hybrid PIC model and from the present analysis in this paper.

\subsubsection{Gas purity}

The gas purity is an intrinsic concern for the EUV scanner for several reasons: outgassing of reticles coming in from ambient, microleaks from load locks and robots, and outgassing from vessel walls (as the delicate scanner system cannot be baked out at high temperature). This can lead to trace gas levels in the $\mathrm{RME}$ of $\mathrm{N}_{2}, \mathrm{O}_{2}, \mathrm{H}_{2} \mathrm{O}$, and volatile hydrocarbons. Even trace levels of these molecules may change the plasma chemistry, driven by higher EUV absorption to form $\mathrm{N}_{2}^{+}$and $\mathrm{O}_{2}^{+} / \mathrm{O}_{2}^{-}$, by efficient proton transfer to form $\mathrm{N}_{2} \mathrm{H}^{+}$and $\mathrm{H}_{3} \mathrm{O}^{+}$, and by hydrogen chemistry to form other carbon/nitrogen-containing ions. ${ }^{33}$

The change in plasma composition might also change the plasma characteristics, as these heavier ions would diffuse out more slowly and would thus show more build-up over pulses. Earlier investigations, using isolated pulses, showed that trace amounts of $\mathrm{N}_{2}$ might indeed affect the plasma composition and chemistry but do not change the ion energy distribution function (IEDF) of the hydrogen ions significantly. ${ }^{60}$ This was confirmed recently on an LPP testrig for regular 50-kHz multipulse mode of operation. No measurable difference in IEDF was observed for an addition of $10^{-2} \mathrm{~Pa}$ of $\mathrm{N}_{2}$ level in 5 to $10 \mathrm{~Pa} \mathrm{H}_{2}$, as shown in Fig. 18.

Still, care should be taken regarding gas purity since the heavier ions may have significantly more impact on materials at the same ion energy, and both oxygen and nitrogen compounds may be chemically active. ${ }^{61,62}$ Also, hydrocarbons and volatile hydrides may have a significant impact already at trace levels since these may be decomposed by the EUV to result in deposition of carbonaceous layers on mirrors ${ }^{63}$ and reticles. ${ }^{64}$

\section{Reticle Mini-Environment}

In the scanner, the EUV-induced plasma is different for different locations within the scanner, since every successive mirror in the optical system absorbs $\sim 30 \%$ of light; ${ }^{65}$ the typical range of plasma parameters at the reticle level is given in Table 2. Furthermore, at the reticle level, the geometry around the beam is severely constrained, with reticle masking blades and other surfaces at close proximity, as shown in Fig. 19. As outlined in Sec. 3.4.4, the typical length of the RME confinement is $\Lambda_{w} \approx 1.5 \mathrm{~cm}$, and only limited build-up of plasma over multiple pulses occurs. The RME dimensions are in the order of the mean-free path length of the electrons and ions, so ions experience only a few collisions, if any. The resulting narrow slits suppress ambipolar plasma diffusion, but fast photoelectrons may travel through these slits to create secondary ionization events up to $\sim 10 \mathrm{~cm}$ from the EUV beam. At this location of the scanner, no 
Table 2 Summary of EUV-induced plasma parameters in RME.

\begin{tabular}{lc}
\hline \hline Parameter & Value \\
\hline Incident EUV power & $50 \mathrm{~W}$ \\
Plasma electron/ion density & $\sim 10^{15} \mathrm{~m}^{-3}$ \\
Ionization degree & $\sim 10^{-4} \%$ \\
Ion flux & $\sim 10^{17} \mathrm{~m}^{-2} \mathrm{~s}^{-1}$ \\
lon energy & $<10 \mathrm{eV}^{-1}$ \\
Radical flux & $\sim 10^{19} \mathrm{~m}^{-2} \mathrm{~s}^{-1}$ \\
\hline \hline
\end{tabular}

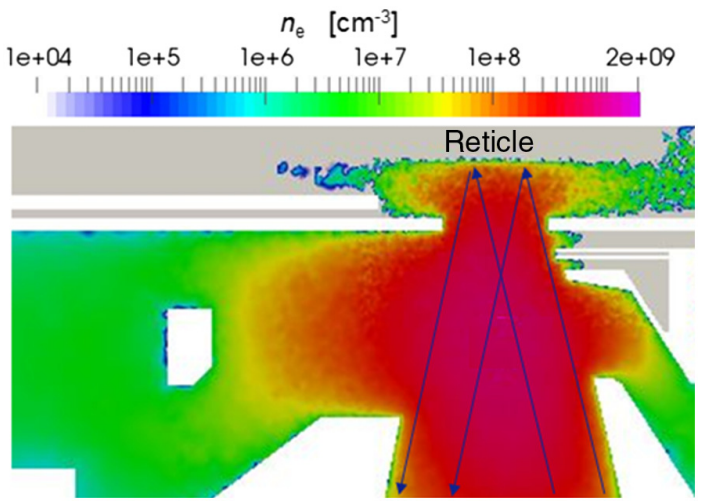

Fig. 19 Schematic of EUV-induced plasma in RME, showing high plasma density in EUV beam (arrows), but also plasma expansion throughout the volume and in between reticle and reticle masking blades (see Fig. 1 for components).

measurements are possible, and we have to rely on the PIC model and the validation thereof as described above.

The reticle itself is a patterned reflector. It is irradiated with an EUV beam from the illuminator and reflects the light back into the projection optics, with a diffraction pattern containing the reticle pattern information. The reticle is floating, with independent conductive backside and frontside layers. ${ }^{66}$ The surrounding surfaces, such as reticle masking blades, uniformity correction blades and other plasma-facing walls are conductive and grounded. As outlined in Sec. 3.4.2, the photoelectric effect is significant in the vicinity of mirrors or reticle, and more electrons are actually generated from the surface by photoemission than by gas ionization. This results in transient positive charging of the reticle frontside to $\sim 30 \mathrm{~V}$ during the EUV pulse, ${ }^{65}$ as shown in Fig. 20. When irradiation stops, the low-energy electrons are reabsorbed by the positively charged surface, which in combination with the electrons from the ionized gas brings the surface to the same potential as the plasma within the first $\sim 1 \mu \mathrm{s}$.

This transient reticle-plasma potential significantly suppresses the peak ion energies toward the reticle in the high-energy phase during and directly after the EUV pulse. ${ }^{30}$ This results in reduced ion energies toward the irradiated surface and enhanced ion energies to the grounded surfaces, as shown in Fig. 21.

Thus, although these surrounding plasma-facing construction and functional materials are not directly irradiated by EUV (although some straylight may be present), the resulting ion and radical load may be significant, and all materials in this area need to be qualified rigorously. 


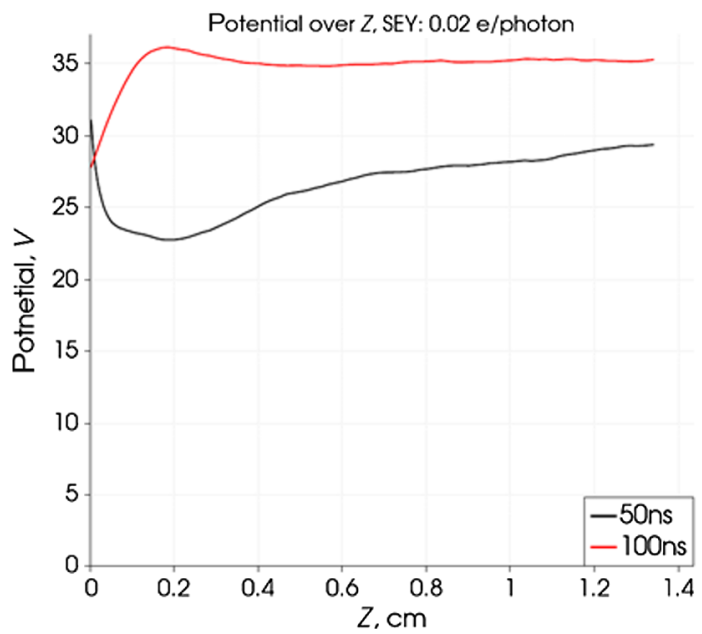

Fig. 20 Modeled impact of SEE /SEY on plasma-wall potential at $50 \mathrm{~ns}$ (black) and $100 \mathrm{~ns}$ (red), showing the initial inverse sheath and subsequent fast transition to classical sheath.

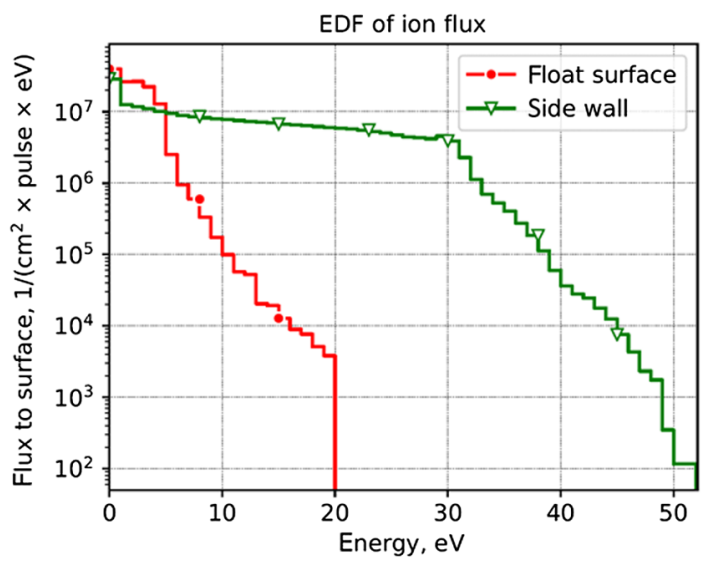

Fig. 21 Modeled ion energy distribution functions for floating reticle surface exposed to EUV (red) and grounded side wall surface close to exposed surface (green).

\section{Offline Emulation of Scanner Plasma}

For studies of plasma-material interactions as well as plasma-particle interactions, it is beneficial to reproduce the EUV-induced plasma conditions inside the scanner with the help of smaller laboratory EUV sources. Typically, laboratory sources have less output power than a commercial LPP EUV source, so setups often employ a significantly smaller spot to reproduce a similar timeaveraged EUV irradiance. Beam spots of $\sim 1 \mathrm{~cm}$ or smaller show pronounced ion diffusion to the sides, reducing the effective ion load to the irradiated spot and increasing the ratio of photons to ions and radicals.

Also, the repetition rate might be quite different. The scanner LPP source operates at $50 \mathrm{kHz}$, while a synchrotron may run at $\sim 0.5 \mathrm{GHz}$ (e.g., PTB Berlin) and a DPP source may run at 1 to $10 \mathrm{kHz}$; this is also reflected in the pulse energies, which are $\sim 10^{5}$ times higher for DPP than for synchrotron for the same focus spot size. These deltas significantly change the balance between the quasisteady-state plasma and the transient peaks, thus resulting in a different IEDF. A synchrotron may be treated as a continuous source and DPP as isolated pulses; scanner LPP is in the middle.

To illustrate this, let us study a model situation in which the laboratory source focuses EUV radiation onto a spot with an area of $0.1 \mathrm{~cm}^{2}$ and a power density of $0.1 \mathrm{~mJ} / \mathrm{cm}^{2}$. Three different types of sources are considered: one with a very high repetition rate of $0.5 \mathrm{GHz}$ (synchrotron), 


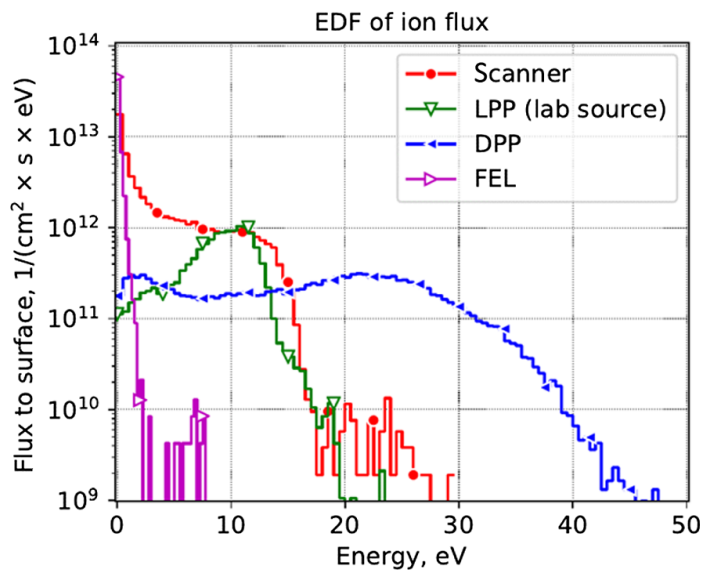

Fig. 22 Ion energy distribution function summed over ion types, around center area of spot $\left(\sim 0.07 \mathrm{~cm}^{2}\right)$.

one with a $50 \mathrm{kHz}$ repetition rate (laboratory LPP), and one with a repetition rate of $5 \mathrm{kHz}$ (DPP). To make the comparison, we used a scanner-like baseline use case with an EUV beam uniformly filling a circle with an area of $10 \mathrm{~cm}^{2}$, using the same EUV irradiance for all sources. Figure 22 shows simulation results for the ion flux to the surface for these four cases. The $0.5-\mathrm{GHz}$ source results in too low ion energies, even if the energy integrated flux in the exposure spot is similar to the baseline case. The $50-\mathrm{kHz}$ source repetition rate results in a similar high energy ion tail as for the scanner case, but the high plasma potential, which is formed during the EUV pulse, causes fast radial expansion of the plasma and reduced ion dose in the exposed area. The case with $5-\mathrm{kHz}$ repetition rate results in high ion energies and further reduction of ions in the exposure spot due to enhanced plasma expansion; also, the $10 \times$ higher peak irradiance during the pulse at this lower frequency might change the plasma-material interaction.

So even if the laboratory source can deliver an EUV irradiance matching to scanner, the experimental setup might still need to compensate for the differences in the ion fluxes from the LPP EUV source. To some degree, these differences may be corrected: for the $0.5-\mathrm{GHz}$ case, the exposed sample could be biased to get the desired energy spectrum, and for the $5-\mathrm{kHz}$ laboratory source, a combination of bias and pressure could be used to tune the spectrum. Also, a combination of EUV source and an inductively/capacitively coupled plasma, and/or a radical source, could be used to achieve the desired ion fluxes and energies.

In addition to the temporal and spatial differences, the spectrum of laboratory setups might be significantly different from the scanner. This might originate from differences in the EUV source (e.g., using Xe instead of Sn or DPP instead of LPP) or from less effective spectral filtering in the ionizing 14 to $80 \mathrm{~nm}$ wavelength range (e.g., using grazing-incidence mirrors instead of normalincidence Bragg mirrors). Even minor amounts of out-of-band light may impact the ion flux and ion energies significantly as the ionizing cross section increases for longer EUV/VUV wavelengths. In addition to the $<100$-nm EUV/VUV wavelengths, the UV wavelength range up to $\sim 200 \mathrm{~nm}$ should be considered in view of surface electrons potentially being released by the photoelectric effect.

The resulting significant discrepancies in plasma characteristics form a major complication in comparing laboratory setups with scanner conditions and put critical requirements on the physical accuracy of the models used for interpretating and translating results.

To circumvent the issues described above, it is recommended to combine independent sources of EUV photons, ions, and radicals to best emulate the scanner plasma in an off-line setup. For example, by combining a focused EUV beam with filament-based radical generation and with biasing of sample and surrounding walls for the ion flux and energies. In this case, the EUV photons are not considered relevant; for example, a combination of fast electrons from an e-beam and lower-energy electrons from an ICP plasma and a hot-filament radical source could also be a good alternative. 


\section{Conclusions}

The EUV-induced hydrogen plasma as used in lithographic scanners has been described in detail, both for single EUV-pulses and for high-frequently pulsed LPP sources. The existing descriptions of more or less open EUV-induced plasma's were extended with a treatment of confinement, with plasma-facing walls at a distance on the order of the mean-free path length of the energetic photoelectrons; it was found that this requires explicit inclusion of the relatively lowenergy SEE from the walls, which may be induced by the photoelectric effect or by SEE by the energetic photoelectrons. The resulting significant deviation from thermal equilibrium requires a bi-Maxwellian treatment for an analytical approximation even after the immediate transients EUV pulse itself.

For a pulsed plasma with a period shorter than the decay time of the plasma, the plasma consists of a quasisteady-state cold background plasma and periodic transient peaks in ion energy and ion flux. This aspect also requires a bi-Maxwellian treatment. In terms of modeling, this means that no assumptions can be made on the electron distribution functions, and a (MonteCarlo) PIC model is needed. We have presented an extension of the PIC model approach to complex 3D geometries and to multiple pulses using a hybrid PIC-diffusion approach.

It was found that plasma confinement and resulting contributions from SEE delay the formation of the plasma sheath and thereby reduce the peak ion energies, to below the sputtering threshold for mirrors and construction materials. This holds both for close confinement around the beam as well as for close proximity to an EUV-mirror or the reticle. As a general design rule, a confinement as close as possible around the beam is recommended to minimize ion energies. Materials with a high SEE may also be beneficial in this respect, but care should be taken that all materials are robust against hydrogen radicals and ion-enhanced chemical reactions with hydrogen. The UV afterglow of EUV generation might last longer than the EUV pulse itself and might thereby frustrate sheath formation for some tenths of a microsecond; this also results in reduced peak ion energies as the energetic electrons cool down fast in the meantime.

Close to the EUV-beam, radicals may have high kinetic energies of $>1 \mathrm{eV}$ and might be in an electronically excited state, both of which increase reaction rates. In addition to enhanced chemical reaction rates, the high-energy radical fraction in a confined EUV-induced plasma is also a concern for many metals as these radicals easily penetrates beyond the surface barrier and may result in high concentrations of absorbed hydrogen and consequently in, e.g., hydrogen embrittlement or blistering, or in the case of coatings, in loss of adhesion and delamination.

Some discrepancies are observed between the modeled energies and the measured energies. The underestimation in the model for the mid-term ion energies, which are measured to be $\sim 2 \mathrm{eV}$, is attributed to the superelastic collision effect not being captured sufficiently in the model. There is also some discussion on the absolute value of the RFEA energy measurements, as the RFEA itself is clearly affected by the photoelectric effect from the EUV pulse in the transient phase. Work has started to install an EQP on the LPP source testrig to verify these aspects of the RFEA.

The observed sensitivity to background hydrogen pressure implies that pressure could be used to tune the ion flux and energies. For a confined plasma, lower pressure results in reduced ion flux and reduced peak ion energies, whereas higher pressure results in increased flux and energies. Care should be taken though as a too low hydrogen pressure might reduce self-cleaning of the mirrors and might increase particle release.

The peculiarities and transients of the scanner hydrogen plasma make it difficult to translate findings from off-line laboratory EUV setups to a scanner. Lower or higher pulse frequencies, as well as small focused spots or different confinement geometries, may change the interplay between photons, ions, and radicals. Deeper understanding of the scanner plasma will allow for better interpretation and translation of findings on off-line setups. It is recommended to explore the use of combined setups to better emulate the EUV plasma, e.g., a combination of ICP plasma with fast electrons from an e-beam and a hot-filament radical source. Also, the photon spectrum of laboratory setups will often be significantly different from the scanner. This might originate from differences in the EUV source (e.g., Xe instead of Sn or DPP instead of LPP) or from different spectral filtering in the ionizing 10 to $80 \mathrm{~nm}$ wavelength range (e.g., using 
grazing-incidence mirrors instead of normal-incidence Bragg mirrors). Also, the UV wavelength range up to $\sim 200 \mathrm{~nm}$ is relevant in view of the possibility of cold surface electrons being released by the photoelectric effect.

In summary, the EUV-induced scanner plasma is instrumental in maintaining high system transmission by preventing carbon contamination and oxidation of the EUV mirrors. Potential side effects, however, are etching of the surrounding construction materials and particle release. The improved understanding of the scanner plasma in recent years has enabled mitigation of these side effects and has helped to ensure that the EUV lithography scanners run reliably in HVM at high source powers. These models and understanding enable plasma-aware design guidelines and testing protocols for future EUV systems to be compatible with increasing source powers.

Looking toward the future, the EUV power will continue to rise to enable throughput improvements in the scanner. The ion flux will scale linearly with increasing EUV pulse energy, whereas the ion energy is independent of this, as all electron populations scale equally with power.

\section{Acknowledgments}

The authors wish to thank the ASML Research and Development teams for Scanner Plasma and Defectivity for scanner testing, fruitful discussions, and general support. We would also like to thank Pavel Krainov, Bogdan Lakatosh, Slava Medvedev, and ISAN for PIC simulations.

\section{References}

1. M. van de Kerkhof et al., "Lithography for now and the future," Solid-State Electron. 155, 20-26 (2019).

2. R. van Es et al., "EUV for HVM: towards an industrialized scanner for HVM NXE3400B performance update," Proc. SPIE 10583, 105830H (2018).

3. M. van de Kerkhof et al., "Advanced particle contamination control in EUV scanners," Proc. SPIE 10957, 109570U (2019).

4. O. Braginsky et al., "Removal of amorphous $\mathrm{C}$ and Sn on Mo: Si multilayer mirror surface in hydrogen plasma and afterglow," J. Appl. Phys. 111, 093304 (2012).

5. J. Beckers, "EUV-induced plasma: a peculiar phenomenon of a modern lithographic technology," Appl. Sci. 9, 2827 (2019).

6. I. Fomenkov, EUV Source Workshop, V. Bakshi, Ed. (2018).

7. F. Torretti et al., "Spectral characterization of an industrial EUV light source for nanolithography," J. Phys. D: Appl. Phys. 53(5), 055204 (2019).

8. G. Wannier, Statistical Physics, chapter 10.2, Dover Publications (1987).

9. N. Gambino et al., "Vacuum ultraviolet spectral emission properties of Ga, In and Sn droplet-based laser produced plasmas," Spectrochim. Acta Part B: At. Spectrosc. 122, 149-156 (2016).

10. M. van de Kerkhof et al., "Spectral purity performance of high-power EUV systems," Proc. SPIE 11323, 1132321 (2020).

11. M. van de Kerkhof et al., "High-power EUV lithography: spectral purity and imaging performance," J. Micro/Nanolithogr. MEMS MOEMS 19, 033801 (2020).

12. A. Heays et al., "Photodissociation and photoionisation of atoms and molecules of astrophysical interest," Astron. Astrophys. 602, A105 (2017).

13. H. Sakaguchi et al., "Absolute evaluation of out-of-band radiation from laser-produced tin plasmas for extreme ultraviolet lithography," Appl. Phys. Lett. 92, 111503 (2008).

14. H. Sakaguchi et al., "Spectroscopy of out-of-band radiation from laser-produced tin plasma of EUV light source," 2006, http://www.ile.osaka-u.ac.jp/zone1/public/publication/apr/ 2006/pdf/3/3.17.pdf.

15. M. Lowisch et al., "Optics for EUV production," Proc. SPIE 7636, 763603 (2010).

16. M. van der Velden, "Radiation generated plasmas: a challenge in modern lithography," $\mathrm{PhD}$ thesis, Technical University Eindhoven (2008). 
17. M. Lieberman, "Principles of plasma discharges and materials processing," 2nd ed., John Wiley \& Sons, Hoboken, New Jersey (2005).

18. T. van de Ven, "Ion fluxes towards surfaces exposed to EUV-induced plasmas," PhD thesis, Technical University Eindhoven (2018).

19. V. Demidov et al., "Nonlocal effects in a bounded afterglow plasma with fast electrons," IEEE Trans. Plasma Sci. 34, 825-833 (2006).

20. V. Godyak et al., "Tonks-Langmuir problem for a bi-Maxwellian plasma," IEEE Trans. Plasma Sci. 23, 728-734 (1995).

21. https://www.impedans.com/semion-single-sensor (2021).

22. T. van de Ven et al., "Analysis of retarding field energy analyzer transmission by simulation of ion trajectories," Rev. Sci. Instrum. 89, 043501 (2018).

23. M. van der Velden et al., "Particle-in-cell Monte Carlo simulations of an extreme ultraviolet radiation driven plasma," Phys. Rev. E 73, 036406 (2006).

24. D. Astakhov et al., "Exploring the electron density in plasma induced by EUV radiation: II. Numerical studies in argon and hydrogen," J. Phys. D Appl. Phys. 49, 295204 (2016).

25. T. van de Ven, "Ion energy distributions in highly transient EUV induced plasma in hydrogen," J. Appl. Phys. 123, 063301 (2018).

26. A. Lipatov, The Hybrid Multiscale Simulation Technology: An Introduction with Application to Astrophysical and Laboratory Plasmas, Springer Berlin Heidelberg, Berlin (2002).

27. J. Samson et al., "Total photoabsorption cross sections of $\mathrm{H}_{2}$ from 18 to $113 \mathrm{eV}$," J. Opt. Soc. Am. B 11, 277-279 (1994).

28. Y. Chung et al., "Dissociative photoionization of $\mathrm{H}_{2}$ from 18 to $124 \mathrm{eV}$," J. Chem. Phys. 99, 885 (1993).

29. H. Kossmann et al., "Photoionisation cross section of $\mathrm{H}_{2}$," J. Phys. B: At. Mol. Opt. Phys. 22, L411-L413 (1989).

30. T. Sharp, "Potential-energy curves for molecular hydrogen and its ions," At. Data Nucl. Data Tables 2, 119-169 (1970).

31. M. van der Velden et al., "Kinetic simulation of an extreme ultraviolet radiation driven plasma near a multilayer mirror," J. Appl. Phys. 100, 073303 (2006).

32. J. Berkowitz, Atomic and Molecular Photoabsorption: Absolute Partial Cross Sections, Elsevier Science (2015).

33. H. Tawara et al., "Cross sections and related data for electron collisions with hydrogen molecules and molecular ions," J. Phys. Chem. Ref. Data 19, 617 (1990).

34. J. Yoon et al., "Cross sections for electron collisions with hydrogen molecules," J. Phys. Chem. Ref. Data 37, 913-931 (2008).

35. T. Oka, "Interstellar $\mathrm{H}_{3}^{+}$," Chem. Rev. 113, 8738-8761 (2013).

36. T. Tabata, "Analytic cross sections for collisions of $\mathrm{H}^{+}, \mathrm{H}_{2}{ }^{+}, \mathrm{H}_{3}{ }^{+}, \mathrm{H}, \mathrm{H}_{2}$, and $\mathrm{H}^{-}$with hydrogen molecules," At. Data Nucl. Data Tables 76, 1-25 (2000).

37. H. Hertz, "Ueber einen Einfluss des ultravioletten Lichtes auf die electrische Entladung," Ann. der Phys. Chem. 267, 983-1000 (1887).

38. R. Day et al., "Photoelectric quantum efficiencies and filter window absorption coefficients from $20 \mathrm{eV}$ to $10 \mathrm{keV}$," J. Appl. Phys. 52, 6965-6973 (1981).

39. B. Yakshinskiy, "Carbon accumulation and mitigation processes, and secondary electron yields of ruthenium surfaces," Proc. SPIE 6517, 65172Z (2007).

40. P. Krainov et al., "Dielectric particle lofting from dielectric substrate exposed to low-energy electron beam," Plasma Sour. Sci. Technol. 29, 085013 (2020).

41. R. van der Horst, "Electron dynamics in EUV-induced plasmas," $\mathrm{PhD}$ thesis, Technical University Eindhoven (2016).

42. N. Balcon et al., "Secondary electron emission on space materials: evaluation of the total secondary electron yield from surface potential measurements," IEEE Trans. Plasma Sci. 40, 282-290 (2011).

43. Y. Lin et al., "A new examination of secondary electron yield data," Surf. Interface Anal. 37, 895-900 (2005).

44. X. Wang et al., "Plasma potential in the sheaths of electron-emitting surfaces in space," Geophys. Res. Lett. 43, 525-531 (2016). 
45. B. Tembe, "Electron thermalization in gases. V. Diatomic molecules $\mathrm{H}_{2}, \mathrm{~N}_{2}$, and CO," J. Chem. Phys. 78, 2030 (1983).

46. J. Beckers et al., "Time-resolved ion energy distribution functions in the afterglow of an EUV-induced plasma," Appl. Phys. Lett. 115, 183502 (2019).

47. H. Ellis et al., "Transport properties of gaseous ions over a wide energy range," At. Data Nucl. Data Tables 17(3), 177-210 (1976).

48. D. Albritton et al., "Mobilities of mass-identified $\mathrm{H}_{3}{ }^{+}$and $\mathrm{H}_{+}$ions in hydrogen," Phys. Rev. 171, 94 (1968).

49. R. Plasil et al., "Advanced integrated stationary afterglow method for experimental study of recombination of processes of $\mathrm{H}_{3}{ }^{+}$and $\mathrm{H}_{3}{ }^{+}$ions with electrons," Int. J. Mass Spectrom. 218, 105-130 (2002).

50. G. Hobbs et al., "Heat flow through a Langmuir sheath in the presence of electron emission," Plasma Phys. 9, 85 (1967).

51. M. Campanell, "Negative plasma potential relative to electron-emitting surfaces," Phys. Rev. E 88, 033103 (2013).

52. M. Larsson et al., "The dissociative recombination of $\mathrm{H}_{3}{ }^{+}-$a saga coming to an end?" Chem. Phys. Lett. 462, 145-151 (2008).

53. P. Dohnal, " $\mathrm{H}_{2}$-assisted ternary recombination of $\mathrm{H}_{3}{ }^{+}$with electrons at $300 \mathrm{~K}$," Phys. Rev. A 90, 042708 (2014).

54. M. van Kampen, internal ASML report (2020).

55. M. Lieberman et al., "Global models of pulse-power-modulated high-density low-pressure discharges," Plasma Sour. Sci. Technol. 5, 145 (1996).

56. M. Osiac et al., "Plasma boundary sheath in the afterglow of a pulsed inductively coupled RF plasma," Plasma Sour. Sci. Technol. 16, 355 (2007).

57. A. Maresca et al., "Experimental study of diffusive cooling of electrons in a pulsed inductively coupled plasma," Phys. Rev. 65, 056405 (2002).

58. M. Mozetič et al., "Recombination of neutral hydrogen atoms on AISI 304 stainless steel surface," Appl. Surf. Sci. 144, 399-403 (1999).

59. I. Mendez et al., "Atom and ion chemistry in low pressure hydrogen DC plasmas," J. Phys. Chem. A 110, 6060-6066 (2006).

60. J. Beckers et al., "Energy distribution functions for ions from pulsed EUV-induced plasmas in low pressure $\mathrm{N}_{2}$-diluted $\mathrm{H}_{2}$ gas," Appl. Phys. Lett. 114, 133502 (2019).

61. P. Phadke et al., "Sputtering and nitridation of transition metal surfaces under low energy, steady state nitrogen ion bombardment," Appl. Surf. Sci. 505, 144529 (2020).

62. P. Phadke et al., "Oxidation and sputtering of transition metals by oxygen ions at steady state: sputtering and radiation enhanced diffusion near sputter threshold," Appl. Surf. Sci. 518, 146143 (2020).

63. J. Hollenshead et al., "Modeling radiation-induced carbon contamination of extreme ultraviolet optics," J. Vac. Sci. Technol. B 24, 64 (2006).

64. Y. Fan et al., "Carbon contamination topography analysis of EUV masks," Proc. SPIE 7636, $76360 \mathrm{G}(2010)$.

65. E. Louis et al., "Multilayer coated reflective optics for extreme UV lithography," Microelectron. Eng. 27, 235-238 (1995).

66. M. van de Kerkhof et al., "Plasma-assisted discharges and charging in EUV-induced plasma," J. Micro/Nanopatterning Mater. Metrol. 20, 013801 (2021).

Mark van de Kerkhof began his career at ODME, developing then-novel DVD mastering, and later worked on deep-UV and immersion technologies for Blu-Ray. In 1999, he joined ASML as a senior designer, working both on DUV and EUV scanners, and was responsible for the technical definition and integration of the NXE:3400B EUV scanner as a product system engineer. He currently directs the EUV research projects. He has authored more than 40 scientific papers and holds more than 80 USA patents.

Biographies of the other authors are not available. 